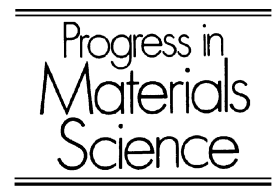

\title{
Multifunctionality of cellular metal systems
}

\author{
A.G. Evans ${ }^{\text {a }}$, J.W. Hutchinson ${ }^{\text {a }}$, M.F. Ashby ${ }^{\text {b, } *}$ \\ ${ }^{a}$ Division of Engineering and Applied Sciences, Harvard University, Cambridge, MA 02138, USA \\ ${ }^{\mathrm{b}}$ Engineering Department, Cambridge University, Cambridge, CB2 1PZ, UK
}

Accepted 23 September 1998

\begin{abstract}
Cellular metals have ranges of thermomechanical properties that suggest their implementation in ultralight structures, as well as for impact/blast amelioration systems, for heat dissipation media and in acoustic isolation. The realization of these applications requires that the properties of cellular metals be understood in terms of their manufacturing constraints and that their thermostructural benefits over competing concepts be firmly established. This overview examines the mechanical and thermal properties of this material class, relative to other cellular and dense materials. It also provides design analyses for prototypical systems which specify implementation opportunities relative to competing concepts. (C) 1999 Elsevier Science Ltd. All rights reserved.
\end{abstract}

\section{CONTENTS}

1. INTRODUCTION

2. SCALING RELATIONS $r$

2.1. Generalities $r$

2.2. Stiffness $r$

2.3. Plastic flow $r$

2.4. Fracture 184

3. MORPHOLOGICAL DEFECTS 186

3.1. Morphological rules 186

3.2. Theoretical results $r$

3.3. Experimental measurements 188

4. HEAT TRANSFER MEDIA $r$

4.1. Concepts 190

4.2. Heat transfer $r$

4.3. Heat fluxes $r$

4.4. Thermal calibrations $r$

4.5. Pressure drop 193

4.6. Trade-off 193

* Corresponding author. Tel.: +44-1223-332635; fax: +44-1223-332662; e-mail: mfa@eng.cam.ac.uk. 
5. MINIMUM WEIGHT STRUCTURES 194

5.1. Structural indices 194

5.2. Stiffness-limited sandwich structures 196

5.2.1. Method of analysis 196

5.2.2. Global minimum 197

5.2.3. Failure limits 199

$\begin{array}{lr}\text { 5.3. Stiffened panels } & 200\end{array}$

5.4. Cylindrical shells $r 202$

$\begin{array}{lr}\text { 5.5. Imperfection sensitivity } & 205\end{array}$

5.6. Other configurations $r$

$\begin{array}{lr}\text { 5.7. Synopsis } & 209\end{array}$

6. ENERGY ABSORPTION 209

6.1. Nondimensional indices $r$

$\begin{array}{lr}\text { 6.2. System comparison } & 210\end{array}$

$\begin{array}{lr}\text { 6.3. Impact } & 211\end{array}$

6.4. Blast amelioration $r$

7. MANUFACTURING STATUS $r 213$

7.1. Gas expansion methods 214

7.2. Hollow sphere methods 216

$\begin{array}{lr}\text { 8. SUMMARY } & 216\end{array}$

$\begin{array}{lr}\text { APPENDIX } & 218\end{array}$

$\begin{array}{lr}\text { REFERENCES } & 220\end{array}$

\section{Nomenclature}

$\begin{array}{ll}2 a: & \text { cell diameter } \\ A_{i}: & \text { bending coefficients (Table 2) } \\ b: & \text { contact radius for hollow spheres } \\ B: & \text { panel width } \\ B i: & \text { Biot number } \\ 2 c: & \text { crack length } \\ C: & \text { heat transfer parameter (19) } \\ d_{:} & \text {diameter of open cell ligaments } \\ d_{\mathrm{b}}: & \text { thickness of buffer plate } \\ d_{\mathrm{c}}: & \text { core thickness } \\ d_{\mathrm{f}}: & \text { face sheet thickness } \\ D: & \text { thickness of foam } \\ E: & \text { Young's modulus of cellular material } \\ E_{\mathrm{f}}: & E \text { for face sheets } \\ E_{\mathrm{s}}: & E \text { for cell walls } \\ G: & \text { shear modulus of cellular material } \\ h_{0}: & \text { local heat transfer coefficient } \\ h_{\mathrm{eff}}: & \text { effective value of } h_{0} \text { for cellular medium } \\ H: & \text { global heat transfer coefficient } \\ I: & \text { inertia } \\ J: & J \text {-integral } \\ J_{\mathrm{c}}: & \text { fracture toughness } \\ k_{\mathrm{s}}: & \text { thermal conductivity of cell wall material }\end{array}$


$k_{\text {eff: }} \quad$ effective thermal conductivity for cellular medium

$k_{\mathrm{f}}$ : thermal conductivity for fluid

$K_{\text {c }} \quad$ fracture toughness of cellular metal

$l: \quad$ heat transfer length

$L$ : $\quad$ length of loaded structure

$m: \quad$ weight per unit area

$N: \quad$ load per unit perimeter

$P: \quad$ load

$Q: \quad$ heat flux

$R_{0}$ : $\quad$ radius of hollow sphere

$R_{\mathrm{s}}: \quad$ radius of shell

$s: \quad$ stiffness

$S: \quad$ stiffness index

$T: \quad$ temperature of solid

$T_{\mathrm{f}}$ : $\quad$ fluid temperature

$U: \quad$ energy absorbed per unit volume $\left(\mathrm{kJ} / \mathrm{m}^{3}\right)$

$U_{\rho}: \quad$ energy absorbed per unit mass $(\mathrm{J} / \mathrm{g})$

$v: \quad$ fluid velocity

w: $\quad$ wall thickness for closed cell material

$W: \quad$ weight of structure

$W_{\mathrm{c}}$ : $\quad$ weight of core

$W_{\mathrm{f}}$ : $\quad$ weight of face sheets

\section{Greek symbols}

$\begin{array}{ll}\alpha: & \text { mechanical property coefficient } \\ \alpha_{1}: & \alpha \text { for closed cell material stiffness (1) } \\ \alpha_{2}: & \alpha \text { for open cell material stiffness (2) } \\ \alpha_{3}: & \alpha \text { for closed cell material yield strength (3) } \\ \alpha_{4}: & \alpha \text { for open cell material yield strength (4) } \\ \beta_{1}: & \text { plastic stretch coefficient (5) } \\ \beta_{2}: & \text { fracture toughness coefficient (6) } \\ \chi: & \text { Biot coefficient (10) } \\ \delta: & \text { displacement upon lateral loading of panel } \\ \eta: & \text { strain hardening parameter (Fig. 8c) } \\ \epsilon: & \text { strain } \\ \epsilon_{\mathrm{c}}: & \text { tensile ductility } \\ \epsilon_{\mathrm{Y}}: & \text { yield strain of face sheets } \\ \epsilon_{\mathrm{D}}: & \text { densification strain } \\ \epsilon_{\mathrm{s}}: & \text { yield strain of dense core material } \\ \phi: & \text { imperfection exponent for buckling } \\ \gamma: & \text { knockdown factor for buckling } \\ \kappa: & \text { kinetic energy } \\ \pi: & \text { load index }\end{array}$




$\begin{array}{ll}\pi_{\mathrm{e}}: & \text { elastic load index } \\ \pi_{\mathrm{p}}: & \text { plastic load index } \\ \rho: & \text { relative density of cellular material } \\ \sigma: & \text { stress } \\ \sigma_{0}: & \text { yield strength of cellular material } \\ \sigma_{\mathrm{p}}: & \text { plateau compression strength } \\ \sigma_{\mathrm{S}}: & \text { yield strength of cell wall material } \\ \sigma_{\mathrm{Y}}: & \text { yield strength of face sheets } \\ \Omega: & \text { density of cell wall material } \\ \Omega_{\mathrm{f}}: & \text { density of face sheet material } \\ \Omega_{\mathrm{g}}: & \text { fluid density } \\ \Omega_{\mathrm{b}}: & \text { density of buffer plate } \\ \psi: & \text { weight index }\end{array}$

\section{Introduction}

Cellular metals have combinations of mechanical, thermal and acoustic properties that provide opportunities for diverse thermostructural implementations [1-9]. The technologies include ultralight structures [10,11], impact absorbers [2,12], heat dissipation media and compact heat exchangers [13,14]. Successful implementation relies not just on their thermomechanical properties, but on additional attributes: low manufacturing cost, environmental durability and fire retardancy [15]. Because of this diversity, multifunctional representation/analysis is an essential element in the engineering strategy [16]. This article integrates basic multifunctionality concepts and complements a Cellular Metals Design Manual [2].

Cellular metals have been available for decades [17,18], but new opportunities are emerging for two reasons: (i) novel manufacturing approaches have beneficially affected performance and cost [3-9]; (ii) higher levels of basic understanding about mechanical, thermal and acoustic properties have been developed [2, 19-23] in conjunction with associated design strategies [2, 10, 11, 24]. These provide an integrated pathway between manufacturing and design.

The stress/strain response exhibited by low relative density cellular metals (Fig. 1) establishes two aspects of their engineering utility: (i) their relatively high stiffness and yield strength achievable at low density [16] creates an opportunity for ultralight structures, with integrally bonded dense face sheets; ${ }^{1}$ (ii) large compressive strains achievable at nominally constant stress (before the material compacts) impart a high energy absorption capacity at force levels relevant to crash and blast amelioration systems $[2,16]$.

\footnotetext{
${ }^{1}$ However, the materials are less stiff and strong than theoretically possible, and much less stiff than honeycomb cores. These issues are brought out later.
} 


\section{COMPRESSIONSTRESSISTRAIN BEHAVIOR}
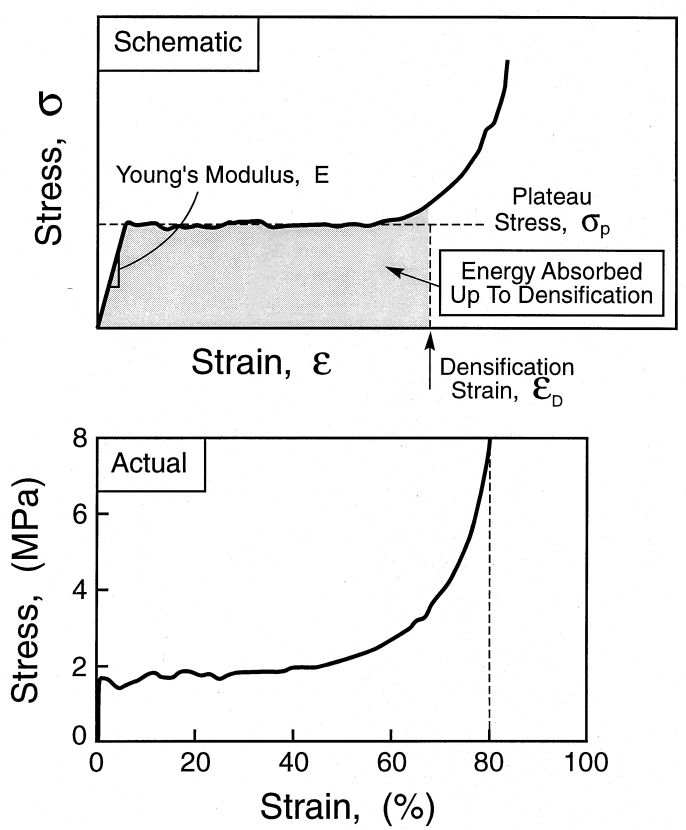

Fig. 1. Ideal and actual stress/strain curves for a closed cell Al alloy (Alporas)

Open cell metals constitute a third opportunity. These materials have thermal attributes that enable applications as heat dissipation media and as recuperation elements [13,14] (Fig. 2). The attributes include the high thermal conductivity of the material comprising the borders, in combination with high internal surface area and propitious fluid transport dynamics. These enable high heat transfer rates that can be used effectively for either cooling or efficient heat exchange.

Cellular metals incorporated within a structure to form sandwich skins can realize systems that achieve mechanical performance and affordability goals at lower weight than competing concepts [11,24]. However, in many other cases, conventional stiffened designs are more weight efficient. An essential step toward implementation comprises structural analysis of prototypical systems. Such analysis identifies sandwich constructions having explicit weight advantages, wherein the cellular core is used to increase the bending stiffness [25]. Weight advantages are found in structures controlled by bending or compression, but not 


\section{CELLULAR HEAT TRANSFER MEDIUM}

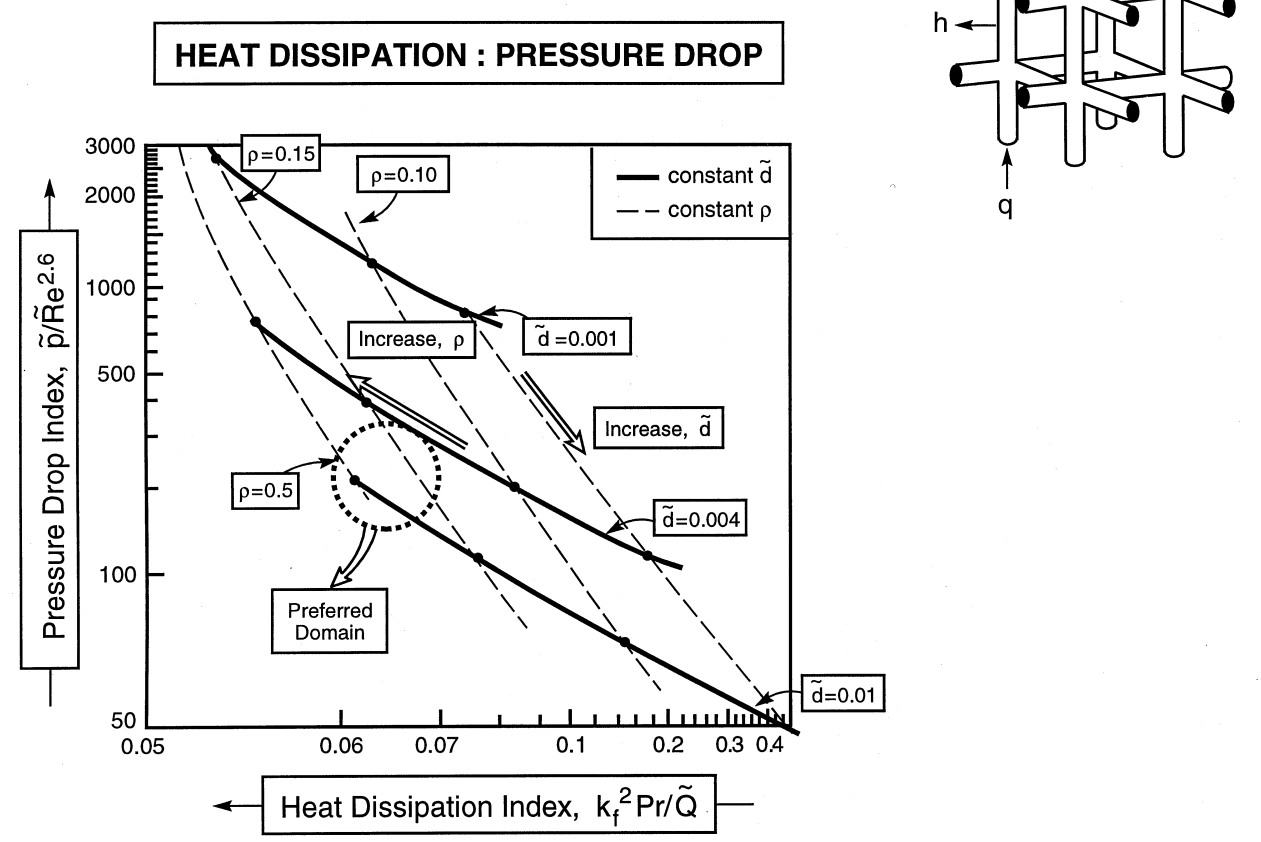

Fig. 2. A schematic of an open cell metal used as a heat dissipation medium, e.g. for cooling high power electronics. Also shown is the trade-off between pressure drop and heat flux, with the preferred material domain indicated. 
in those dominated by tension. ${ }^{2}$ Among such structural elements, some are stiffness limited, while others are strength limited.

The benchmarks for comparison with sandwich skin construction comprise [10,11,24,27]: (i) stringer-stiffened panels or shells; (ii) honeycomb panels; and (iii) hollow tubes. Through decades of development, all three have been optimized and provide performance targets that are difficult to supersede. Often the benefits of cellular metal systems derive from an acceptable structural performance combined with lower costs or greater durability than competing concepts. For example, honeycomb panels comprising polymer composite face sheets with Al honeycomb core are particularly weight efficient: they can never be superseded by cellular metal construction strictly on a performance basis. However, such honeycombs have durability problems associated largely with water intrusion and they are relatively expensive [28]. They are also highly anisotropic and costly to configure as cores for curved structures. Cellular metal construction can become competitive on a performance/durability/affordability basis, particularly for shell structures and geometrically complex panels. Elaboration on these and other considerations comprises one theme of this article.

Another attribute that enables cellular systems to compete favorably with presently available alternatives is their shape flexibility. That is, several of the manufacturing methods [3,4] enable hollow thin-walled configurations with arbitrary geometry to be filled with cellular metal. This process provides a light/ stiff system with broad-ranging geometric complexity that cannot be duplicated using either honeycomb core or stiffeners. A further benefit comprises an appreciable increase in the natural frequency of vibration. This feature can be used for noise suppression

Structures that absorb energy have two dominant properties $[1,2]$ : the energy per unit mass, $U_{\rho}$ (in $\mathbf{J} / \mathrm{g}$ ), and the stress at which this energy is absorbed, $\sigma_{\mathrm{p}}$ (Fig. 1). High energy absorption is required at a predictable and uniform $\sigma_{\mathrm{p}}$. The latter ensures that the force transmitted remains below a critical level that upon impact/blast might otherwise cause structural damage. The former governs the foam thickness needed to absorb the kinetic energy. This crash/blast amelioration function can be realized in three ways: (i) with foam; (ii) with thin-walled tubes; or (iii) with sandwich tubes.

The metrics governing implementation depend on thermomechanical and structural indices, which scale the performance. The coefficients on these indices establish limits for the properties of ideal materials, against which actual materials can be benchmarked. These relations are examined in Section 2. Differences between the ideal and actual performance of cellular metals are addressed in Sections 3 and 4, which examine the mechanical and thermal properties, respectively. Ultralight structures are explored in Section 5 and impact/blast

\footnotetext{
${ }^{2}$ Note that in aircraft deign for example, more than half of the structure is limited by its bending or compressive performance [26].
} 
amelioration systems in Section 6. Finally, manufacturing developments since the last review [17] are summarized in Section 7.

\section{Scaling relations}

\subsection{Generalities}

Mechanical property scaling relations have been established by Gibson and Ashby in their comprehensive text of Cellular Solids [1]. Results pertinent to metals are emphasized here [2]. Distinctions are made between open and closed cell materials which are inherently different. Responses are fundamentally related to bending and stretching deformations [1]. Cellular elements that allow bending are subject to high local stresses that cause the system to be compliant and have low yield strength. Conversely, when the cell walls stretch without bending, the system is stiff and has high strength.

Various high performance cell morphologies that minimize bending deformations have been conceptualized [14,23,30]. Three examples include: periodic tetrakaidecahedra [19], close-packed-bonded spheres [23] and truss structures [30]. Most commercially available materials have inferior mechanical properties.

Ideally, plastic yielding and collapse would occur simultaneously [1] resulting in a distinct yield strength coincident with a plateau flow stress, designated $\sigma_{\mathrm{p}}$. In commercial materials, yielding and collapse are not coincident [22,31]. Local yielding initiates at cell nodes almost immediately upon loading, followed by rapid strain hardening. Accordingly, the elastic domain is confined to very small strains $(<0.1 \%)$. At this stage, narrow deformation bands often form and extend across the test configuration (Fig. 3). Thereafter, a peak develops with subsequent oscillation of the stress about a nominal plateau. The peak is governed by plastic collapse, typically within one of the previously deformed deformation bands [31]. For expediency, the peak stress is defined as the yield strength, $\sigma_{\mathrm{p}}$ (Fig. 1). Unloading measurements are preferred for determining the elastic properties (Fig. 3).

\subsection{Stiffness}

Closed cell structures establish upper limits on stiffness. At the low relative densities $\rho$ of present interest, the Young's modulus, $E$, of such structures scales as $[1,19,20,23,29]$ :

$$
E / E_{\mathrm{s}}=\alpha_{1} \rho
$$

where $E_{\mathrm{s}}$ is the modulus of the solid material comprising the cell walls. The coefficient $\alpha_{1}$ depends on the geometric arrangement of cells. Honeycombs are anisotropic, with $\alpha_{1} \approx 1$ for longitudinal loading. In the transverse direction, the 

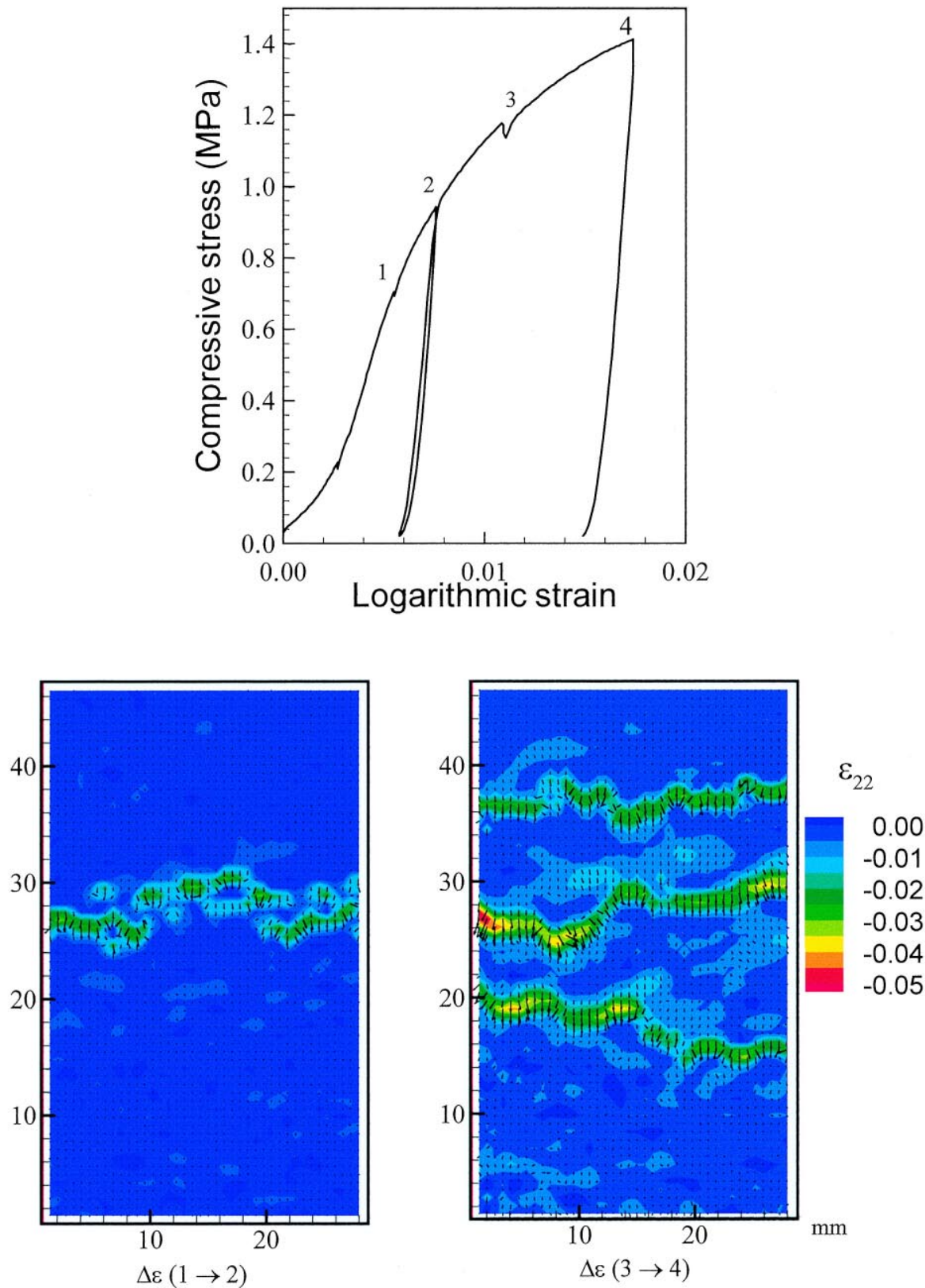

Fig. 3. Strain maps obtained for a closed cell Al material (Alporas) subject to uniaxial compression. Incremental strains $\Delta \epsilon$ between the levels indicated on the stress/strain curve are presented. The maximum principal strain $\epsilon_{22}$ is shown and the vectors indicate the displacements. Note that virtually all of the strain occurs in the narrow deformation bands. Unloading is used to measure the modulus. 
stiffness is considerably lower. It can be represented by [1]:

$$
E / E_{\mathrm{s}}=\alpha *_{1} \rho^{3}
$$

where $\alpha_{1}^{*}$ is of order unity.

For isotropic closed cell metals, ideally $\alpha_{1} \approx 1 / 3$. Numerical results $[19,20,23,29]$ indicate magnitudes quite close to this: (i) for tetrakaidecahedra, $\alpha_{1}=0.35$, with a weak dependence on the distribution of material between the borders and the walls $[19,20]$ (Fig. 4); (ii) for thin-walled spheres, high stiffness can also be
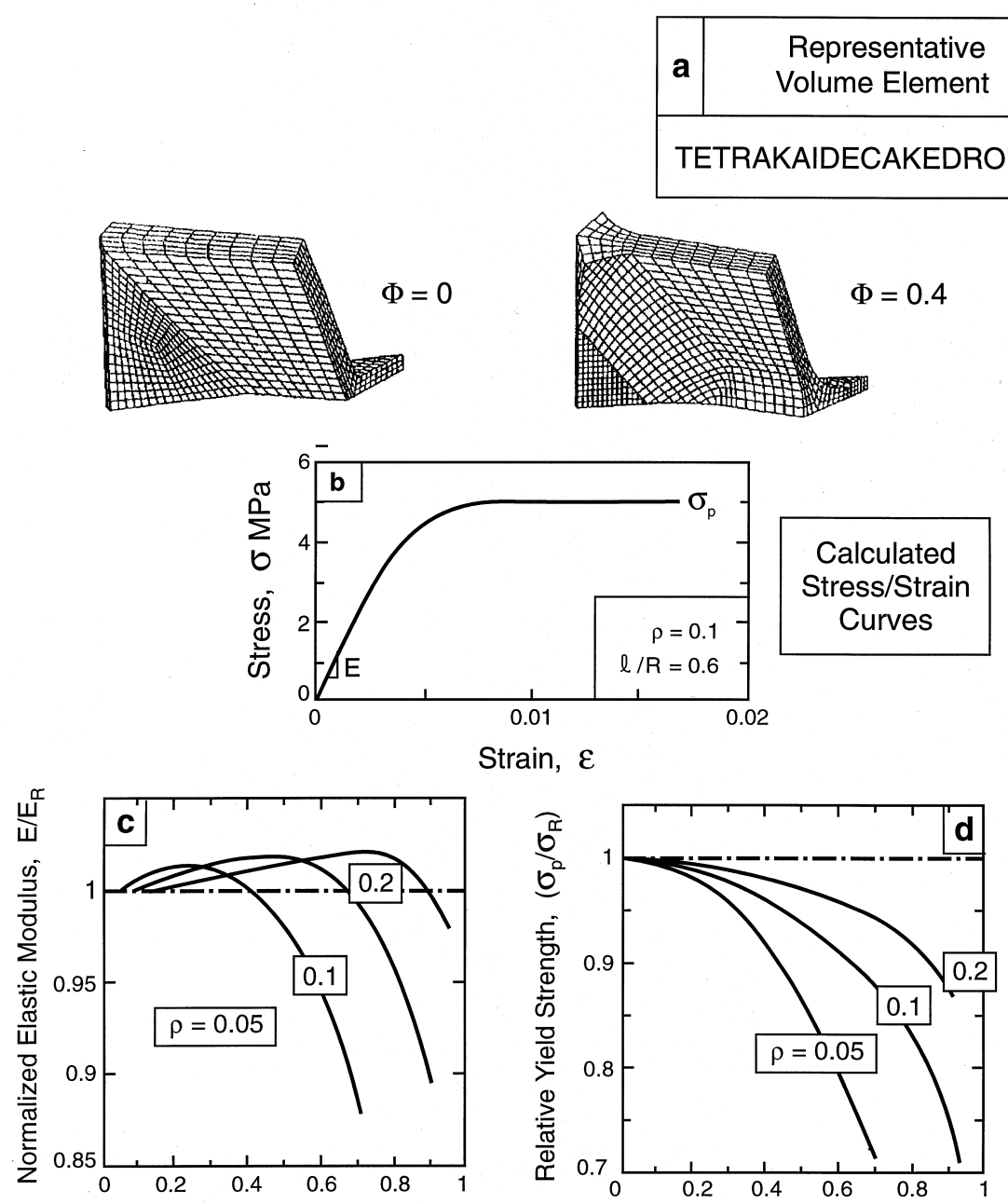

Fraction of Solid in the Plateau Borders, $\Phi$

Fraction of Solid in the Plateau Borders, $\Phi$

Fig. 4. Calculated effects of the distribution of material on: (a) the Young's modulus; and (b) the yield strength of a periodic cell structure comprised of tetrakaidecahedra. Here $E_{\mathrm{R}}$ and $\sigma_{\mathrm{R}}$ are reference values that apply when $\Phi=0[19,20]$. 
obtained $\left(\alpha_{1}=0.35\right)$, but only with f.c.c. packing and when the contact radius is relatively large $b / R_{0}=0.5$. For simple cubic packing it is lower. It reaches, $\alpha_{1}=0.19$ when $b / R_{0}=0.5$, but only $\alpha_{1}=0.04$ when $b / R_{0}=0.1$ (Fig. 5).

Open cell solids, unless specially configured, are susceptible to bending, causing their stiffness to be relatively low and subject to the scaling [1],

$$
E / E_{\mathrm{s}}=\alpha_{2} \rho^{2}
$$

where $\alpha_{2}$ is about unity.

Commercially available closed cell metals have stiffness lower than Eq. (1) $[16,19-22]$. The knockdown factors on are found to range from 2 to 50 (Fig. 6 and 7a). The knockdown effect arises because of morphological defects
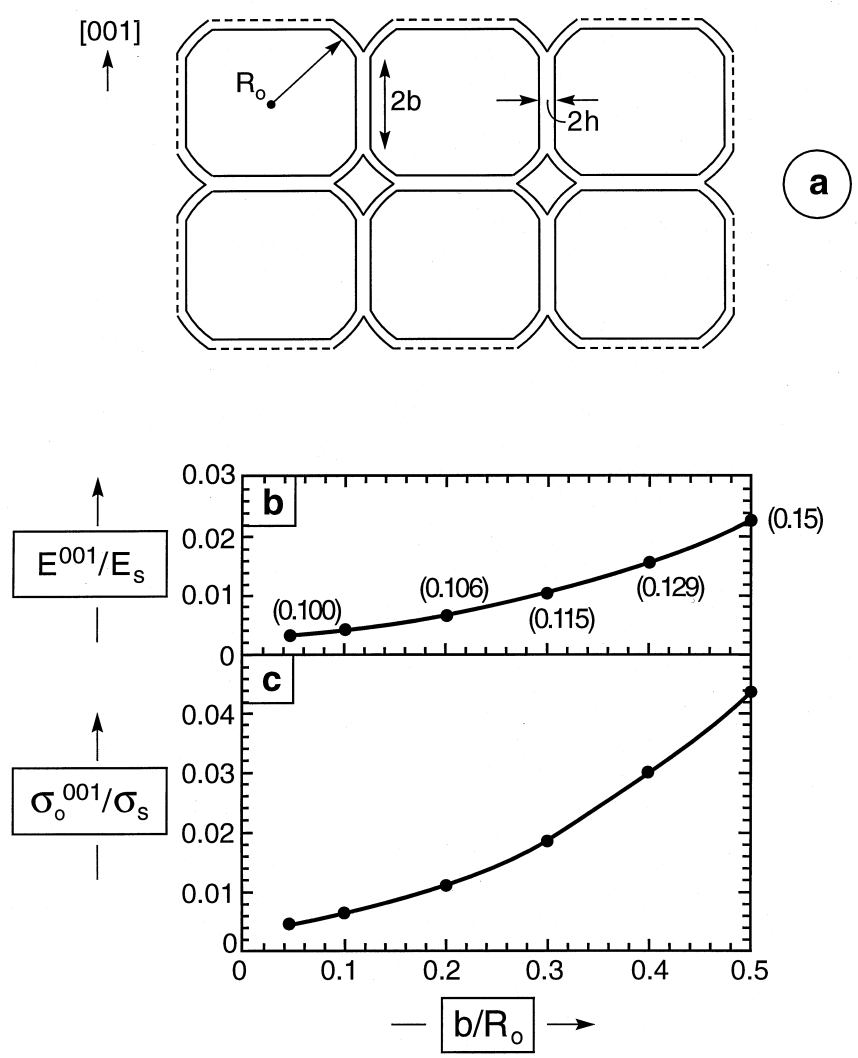

(0.15)

Fig. 5. A simple-cubic packing of hollow spheres with a contact radius, $b$. The Young's modulus and yield strength determined along [001] as a function of $b / R_{0}$ are shown. In the calculations, the relative density change as the contact radius increases. The consequent relative densities are indicated in parenthesis against the results. 


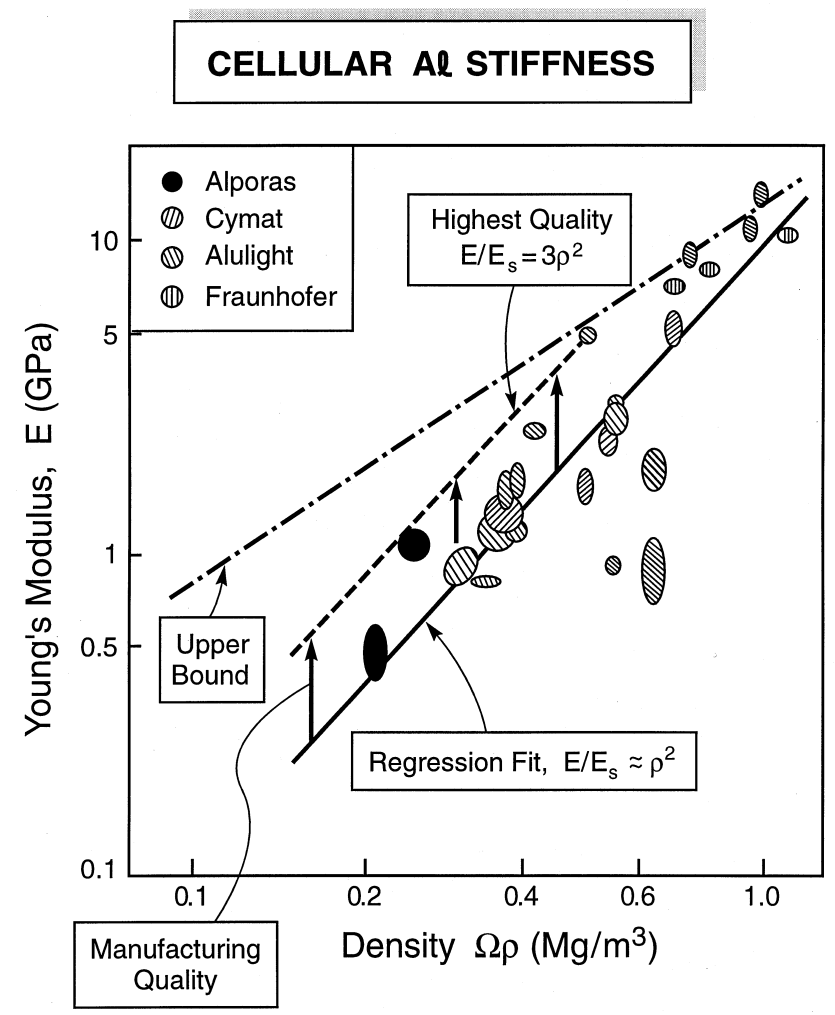

Fig. 6. Stiffness data for Al alloys abtained from CMS software [6].

that induce bending and buckling deformations. The nature of these defects is elaborated in Section 3. Moreover, the totality of available data for closed cell A1 foams (Fig. 6) is more comprehensively represented by (2), rather than (1), with $\alpha_{2}$ ranging from $\backsim 3$ for the higher quality, low density material $\left(<0.5 \mathrm{Mg} / \mathrm{m}^{3}\right)$ to $\sim 1 / 2$ for inferior materials. This phenomenological scaling has utility in the analysis of minimum weight structures, as elaborated in Section 5.

Commercial open cell metals with regular cells have unloading modulus wellrepresented by Eq. (2) (Fig. 7a) [16,19]. In this case, some higher stiffness morphologies have been identified [30,32]. These include hollow border materials made by depositing onto a reticulated polymer foam, subsequently eliminated by pyrolysis. The greater stiffness arises because the bending resistance of the hollow borders exceeds that for their dense counterparts [32]. Various open truss structures can also realize high stiffness [30].

Elastic Poisson ratio measurements indicate a magnitude similar to dense metals $(v \approx 1 / 3)$ [1], such that their shear modulus $G$ is related to their Young's modulus by: $G \approx(3 / 8) E$. The behavior becomes more complicated once yielding has occurred. 


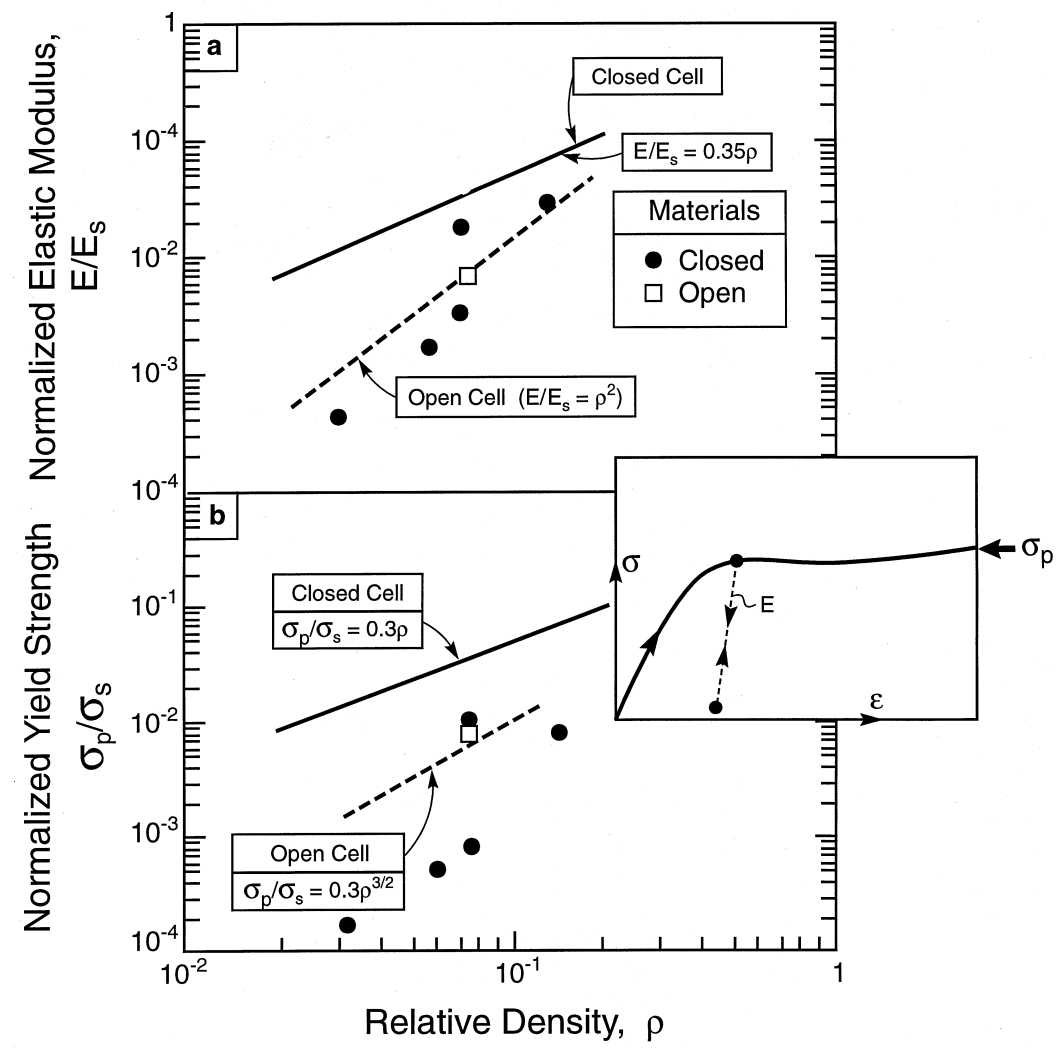

Fig. 7. Comparison of ideal and actual compressive mechanical properties for cellular Al materials: (a) Young's modulus; and (b) yield strength.

\subsection{Plastic flow}

The inelastic properties of cellular metals have not been as extensively studied as their stiffness. Accordingly, the scaling relations remain to be substantiated. Numerical simulations for various cell configurations (Fig. 4a) indicate a negligibly small elastic region, because of localized yielding, followed by rapid strain hardening (even when the base material is perfectly plastic) and then a stress maximum $\sigma_{0}{ }^{3}{ }^{3}$ The available theoretical results for closed cell systems suggest a linear dependence on the density [1, 20,23, 29]:

$$
\sigma_{0} / \sigma_{\mathrm{s}}=\alpha_{3} \rho
$$

where $\sigma_{\mathrm{s}}$ is the yield strength of the material comprising the cell borders. Results

\footnotetext{
${ }^{3}$ Here $\sigma_{0}$ and $\sigma_{\mathrm{p}}$ are taken to be equivalent, since experimentally, initial yielding is ill-defined.
} 
for the periodic tetrakaidecahedron (Fig. 4b), indicate that $\alpha_{3} \approx 0.3$. But now, $\alpha_{3}$ is significantly reduced upon distributing more of the material from the walls within the borders [19]. Results for bonded spheres with simple cubic packing [23] (Fig. 5) reveal that $\alpha_{3}$ approaches 0.3 provided that the contact radius is relatively large, $\left(b / R_{0}=0.5\right)$. The yielding of open cell materials is limited by the bending stresses induced at the nodes, leading to the scaling [1]:

$$
\sigma_{0} / \sigma_{\mathrm{s}}=\alpha_{4} \rho^{3 / 2}
$$

where the coefficient, $\alpha_{4} \approx 0.3$.

Based on estimates of $\sigma_{\mathrm{s}}$ made using microhardness data, the knockdown factor on $\alpha_{3}$ for commercial closed cell $\mathrm{Al}$ alloys is found to range from 4 to 100 [16, 20, 22, 31] (Fig. 7b). The morphological defects discussed in Section 3 are responsible. A phenomenological representation based on Eq. (4) may again apply and have utility for sandwich panel analysis. Commercial open cell materials seemingly satisfy Eq. (4) with essentially no knockdown on $\alpha_{4}[16,19]$ (Fig. 7b).

\subsection{Fracture}

The propagation of cracks through cellular metals is a diffuse, stochastic process because of the spatial variability in the morphologies and properties of the cell walls $[21,33]$. Small cracks, fully contained within a single wall of a closed cell material are the most explicitly defined (Fig. 8a). They are analogous to cracks in thin ductile sheets and propagate at a critical energy release rate, or $J$-integral [21]:

$$
J_{\mathrm{c}} / \sigma_{\mathrm{s}} w=\beta_{1} \rho
$$

where $w$ is the cell wall thickness and $\beta_{1}$ is a plastic stretch coefficient for the wall material. For one commercial Al alloy system, $\beta_{1}$ was found to be in the range $1 / 2$ to 1 [21], dictated by intermetallic particles which limit the plastic stretch.

Converting to stress intensity factors for cells subject to small scale yielding and using Eq. (1) along with the relationship between $w$, the cell diameter, $2 a$, and $\rho$ [1] gives:

$$
K_{\mathrm{c}} /\left[E_{\mathrm{s}} \sigma_{\mathrm{s}} a\right]^{1 / 2}=\beta_{2} \rho^{3 / 2}
$$

where $\beta_{2}=\sqrt{1.2 \alpha_{1} \beta_{1}}$.

In practice, small cracks develop following fully plastic conditions in the cell walls [21]. In this case, the cracks are subject to a $J$-integral [34]:

$$
J / \sigma_{\mathrm{s}} c=\eta \epsilon \rho
$$

where $\eta$ is a function of the strain hardening coefficient for the cell wall material, $N$ (Fig. 8c) and $\epsilon$ is the applied tensile strain. Crack growth thus occurs at a critical strain, $\epsilon_{\mathrm{c}}$, when $J$ in Eq. (7) reaches $J_{\mathrm{c}}$ in Eq. (5):

$$
\epsilon_{\mathrm{c}}=\beta_{1} w / \eta c
$$




\section{CRACK GROWTH}

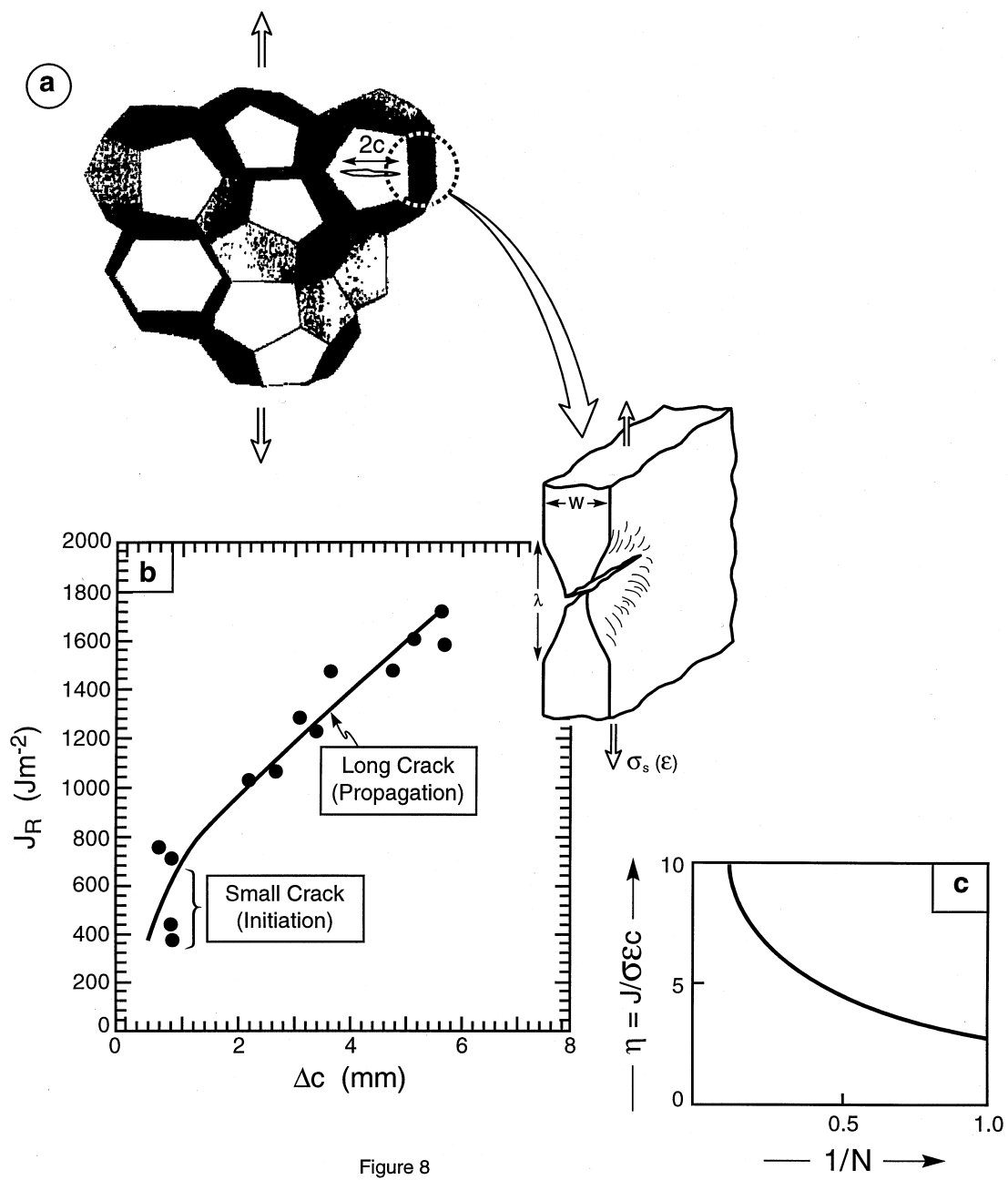

Fig. 8. Crack growth characteristics for an open cell material: (a) a small crack within a cell wall, (b) a resistance curve for a large crack, (c) effect of strain hardening on the $J$-integral coefficient in Eq. (7).

For example, a $1 \mathrm{~mm}$ crack in the cell wall would extend at a strain of $\sim 1 \%$ : of order the measured tensile ductility [21].

Larger cracks become more diffuse, resulting in intact cell walls in the wake [29]. The associated bridging tractions lead to resistance-curve behavior, with large tearing modulus [21,33] (Fig. 8b). 


\section{Morphological defects}

Most commercially available cellular metals, unlike some of their polymer counterparts, do not achieve the properties anticipated by Eqs. (1)-(4). The knockdown factor on these limits ranges from 2 to 100 [2, 19-22] (Fig. 7). Elimination of this knockdown, while not always important [11,24], can sometimes be crucial to the realization of performance advantages, particularly in strength-limited, lightweight structures (Section 5). Various hypotheses have been made regarding the 'defects' that diminish the properties [1-23, 35, 36]. These are now being systematically explored by combining both experimental and theoretical strategies, motivated by the potential to eliminate the most deleterious 'defects' through process control strategies.

\subsection{Morphological rules}

The degrading effects of large bending moments and of low relative density suggest the following four 'rules' about morphological defects.

(i) Closed cellular structures that have straight walls and borders with uniform thickness should exhibit stiffnesses and strengths approaching the limiting values expressed by Eqs. (1) and (3) [20,23,29]. Accordingly, the presence of any features that depart from this rule might degrade the properties. In principle, many such features are possible and, indeed, are found in cellular metal structures [20-22]. They include: curved and wrinkled cell walls, thin or missing walls and high relative density domains (or inclusions). The challenge comprises the quantitative determination of the severity of these features. Progress toward this objective is addressed below.

(ii) The cell size distribution is not a dominant factor. Closed cell materials having essentially straight walls and equiaxed cells can have a relatively wide cell size range but still exhibit properties similar to those for materials with periodic, uniform cells [23,29]. This 'rule' provides a rationale for interpreting observations of morphological defects. 4

(iii) Defects that degrade the elastic properties must normally be present with relatively high volume fraction, $f$. (One caveat is that nonlinear elastic phenomena, such as cell wall buckling, can lead to property degradation at lower $f$.) Composite theory dictates a knockdown factor on stiffness of order, $(1-f)^{-1}$, indicating the need to emphasize only high volume fraction defects. Moreover, it highlights one of the problems in theoretical approaches for quantifying stiffness degrading defects. Namely, when introduced into cells with periodic boundary conditions, defects are necessarily present with high

\footnotetext{
${ }^{4}$ One proviso is that regions with small wall thickness to cell diameter (low local density) may be susceptible to premature yielding. Accordingly, it is important to address and understand local density variations.
} 
spatial frequency. Only by creating a model comprising many cells can the influence of defects with lower spatial frequency be explored.

(iv) Yielding initiates within small domains of spatially correlated defects. These correlated defects enable formation of a band of plastic deformation that spreads across the material [22,31]. An analogy would be compressive kink bands in fiber composites, which develop in accordance with stochastic mechanisms [40]. The corollary of (iv) is that, unlike elastic properties, defects present in small volume fractions are likely to influence yielding.

\subsection{Theoretical results}

Performing theoretical work on morphological defects is challenging, especially for closed cell materials. It is essential to use three-dimensional (3D) models to include the membrane effect, but, it is restrictive to use periodic boundary conditions, because the effect of morphological defects is greatly exaggerated. Leaving out the possibility of a model that combines the $3 \mathrm{D}$ behavior with a sparse population of defects, the approach has been to gain insight from cell calculations. The eventual goal would be to introduce these results into an averaging scheme, such as the self-consistent method, in order to simulate the overall properties. Subject to these provisos, the following calculations have provided insight.

Calculations with periodic boundary conditions have illustrated two effects:

(i) The distribution of material between the walls and the borders does not have an appreciable effect on the stiffness [20] (Fig. 4a). That is, upon thinning the walls (uniformly) and relocating the material at the nodes, the stiffness does not diminish until the walls become thin relative to the cell diameter. This insensitivity arises because bending effects are resisted by material placed at the nodes, thereby counteracting the reduction in membrane stiffness.

(ii) Cell wall curves and wiggles cause dramatic reductions in stiffness and yield strength [20,23]. It remains to quantify their role in a non-periodic structure. Calculations in 2D with nonperiodic cells [39] (Fig. 9) have indicated that missing cell walls markedly diminish the yield strength. By inference, thin cell walls would have a similar effect.

\subsection{Experimental measurements}

The deformations of cells have been monitored using two principal methods: (i) surface deformations are followed by optical microscopy [22,31] (Fig. 3); and (ii) internal cell deformations are reconstructed by using X-ray computed tomography (Fig. 10, circled cell at left) [22] (CT-scan). In the latter, the deformation of 


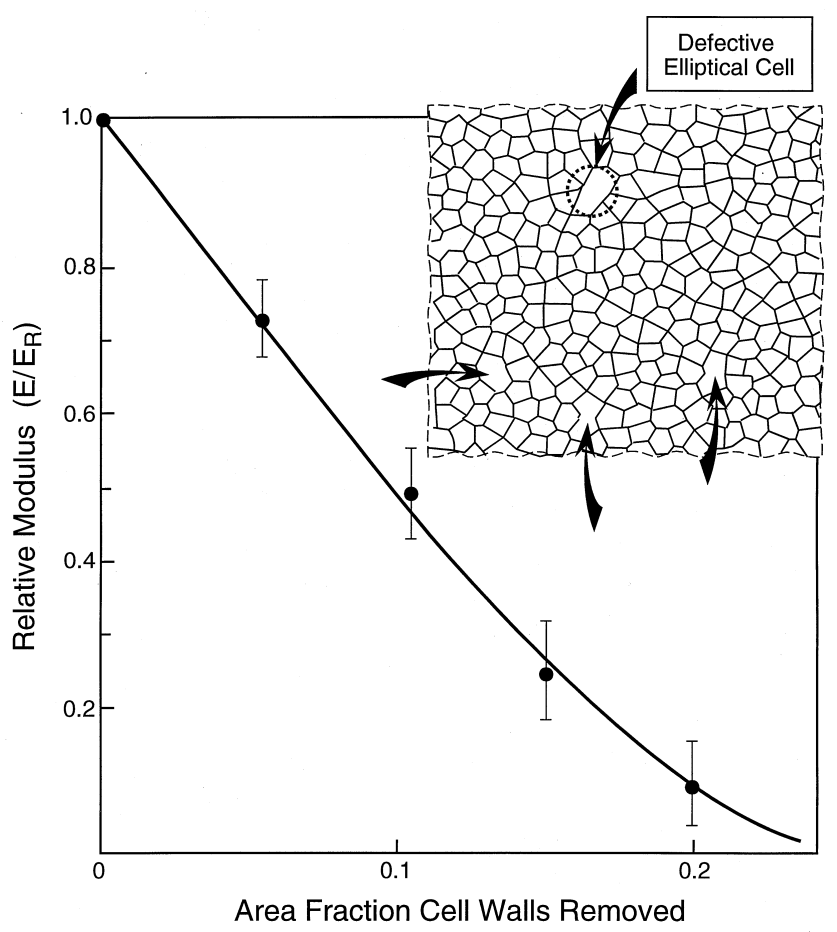

Fig. 9. Effect of missing cell walls on the elastic modulus of a 2D cellular solid [39]. Note the types of defect generated. In the calculations, the relative density changed as the area fraction of missing walls increased. The range refers to the differences found among several simulations. $E_{\mathrm{R}}$ is again the reference value when there are no defects. The reduction in relative yield strength was calculated to be almost identical.

defective cells fully constrained by all of their neighbors can be systematically monitored, enabling the principal morphological defects to be catalogued.

Strain mapping methods (Fig. 3) vividly demonstrate that yielding is heterogeneous and occurs within bands about one cell diameter in width at stresses of order one-third the plateau strength. Moreover, these bands intensify and their number density increases as the stress elevates, until a peak is reached [31]. At the peak, plastic collapse occurs in one of the deformation bands. Each subsequent stress oscillation involves plastic collapse in successive bands.

X-ray results (Fig. 10) have been instrumental in establishing two salient aspects of yielding within the deformation bands [22]. In accordance with 'rules' (i) and (ii), equiaxed cells resist yielding, almost regardless of their size. The corollary is that large cells, if equiaxed, are not the source of the knock-down factor. Consistent with 'rules' (i) and (iv), elliptical cells with their long axis normal to the loading direction are prevalent within deformation bands, regardless of size. Such cells, in cross-section, typically have T-shaped nodes with large entrained angles 


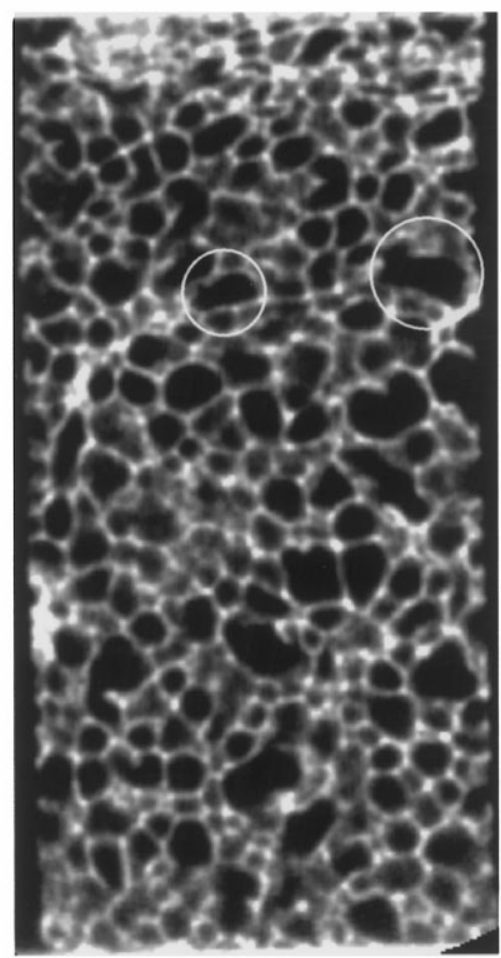

a

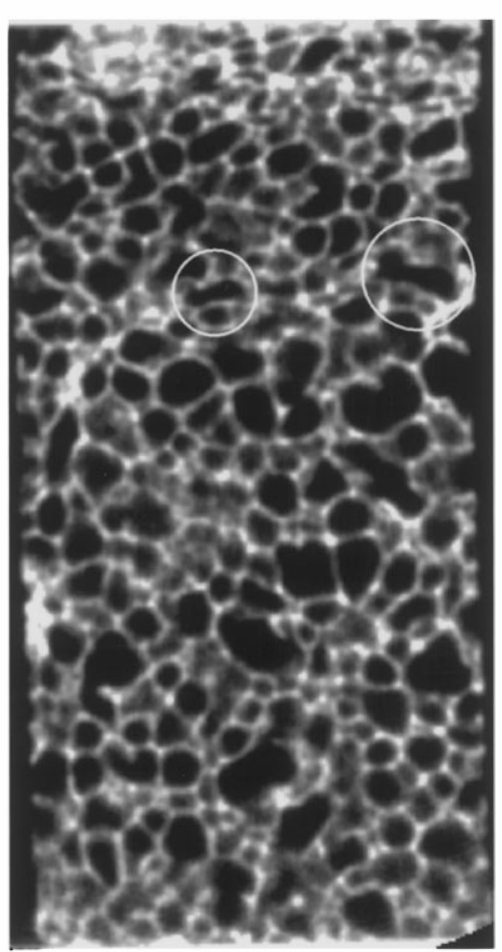

$\mathrm{b}$

Fig. 10. X-ray CAT scan images of cells before and after application of a compressive strain [22]. The circled regions identify those cells located within the deformation band that buckle upon straining. Note that the axial strain can be determined from the shortening of the top.

(Fig. 10, circled cell at left). Such nodes are subject to appreciable bending moments. The inference is that cell ellipticity results in bending effects that reduce the yield strength. A further, unsubstantiated inference is that elliptic cells, if present with sufficient spatial frequency (iii), would also diminish the stiffness. Accordingly, the following assertions are made about morphological defects:

(a) large equiaxed cells are benign;

(b) cell ellipticity is detrimental, particularly for yielding;

(c) cell wall wiggles weaken the material.

However, weakening can be expected from several other defects. Full quantification of all defects and their consequent elimination through processing remains a challenge. 


\section{Heat transfer media}

\subsection{Concepts}

The thermal conductivity of cellular metals is quite large compared with their polymer and ceramic counterparts [1]. Moreover, their thermal diffusivity is comparable to that for dense metals $[2,16]$. These materials are thus of little interest for thermal insulation. Instead, advantage can be taken of their high thermal diffusivity for use as heat exchange media $[13,14]$. In order to calibrate the heat transfer capabilities of cellular metals and, accordingly, extrapolate into new domains, a basic model is needed. There are several options. One, consistent with measurements, regards the cellular medium as a variant on a bank of cylinders. That is, the spatial variations in the temperatures of the solid and fluid have similar forms for the cellular metal medium and the cylinders, but the coefficients differ. Accordingly, proportionality constants are needed to represent the effective thermal conductivity, $k_{\text {eff }}$, and the effective local heat transfer coefficient, $h_{\text {eff. }}$.

A trend toward higher heat dissipation is established, as either the ligament diameter $d$ becomes smaller or the relative density $\rho$ increases. This trend reflects the higher internal surface area as $d$ decreases and the greater heat conduction cross-section as $\rho$ increases. However, this effect is countermanded by an increased drop in the pressure needed to force the fluid through the medium. This pressure drop tends to increase as the surface area to volume ratio increases. Accordingly, there is an optimum cell structure that depends explicitly on the application and its specifications.

\subsection{Heat transfer}

Heat dissipation subject to a transversely flowing fluid (Fig. 2) is characterized by a global heat transfer coefficient, $H$. A model relates $H$ to the features of the medium, through the effective parameters that (once calibrated) enable the thermal behavior to be predicted over a range of cellular material characteristics. The bank of cylinders analogue gives [14, 37-39]:

$$
H=2 k_{\text {eff }} \rho \chi_{\text {eff }} \tanh \left[2 \chi_{\text {eff }} D\right]
$$

where $D$ is the cellular material thickness and

$$
\chi_{\mathrm{eff}}=\sqrt{B i_{\mathrm{eff}}} / d
$$

with $d$ being the diameter of the metal ligaments. The Biot number, $B i$, is a nondimensional measure of the combined effects of conduction along the cell borders and heat transfer into the fluid [13,37-39]. Its effective value is:

$$
B i_{\text {eff }}=h_{\text {eff }} d / k_{\text {eff }}
$$

The challenge is to characterize $k_{\text {eff }}$ and $B i_{\text {eff }}$ in such a manner that effects on $H$ of 
the three material variables $(k, d$ and $\rho$ ) can be predicted. The approach is as follows:

(i) A functional form for $B i$ is chosen.

(ii) Infrared imaging is used to map the distribution of temperature in the cellular metal [13] (Figs. 2 and 11). The same system is used with probes to determine the fluid temperatures.

(iii) The bank of cylinders model is used to simulate the temperature distributions [14].

a)

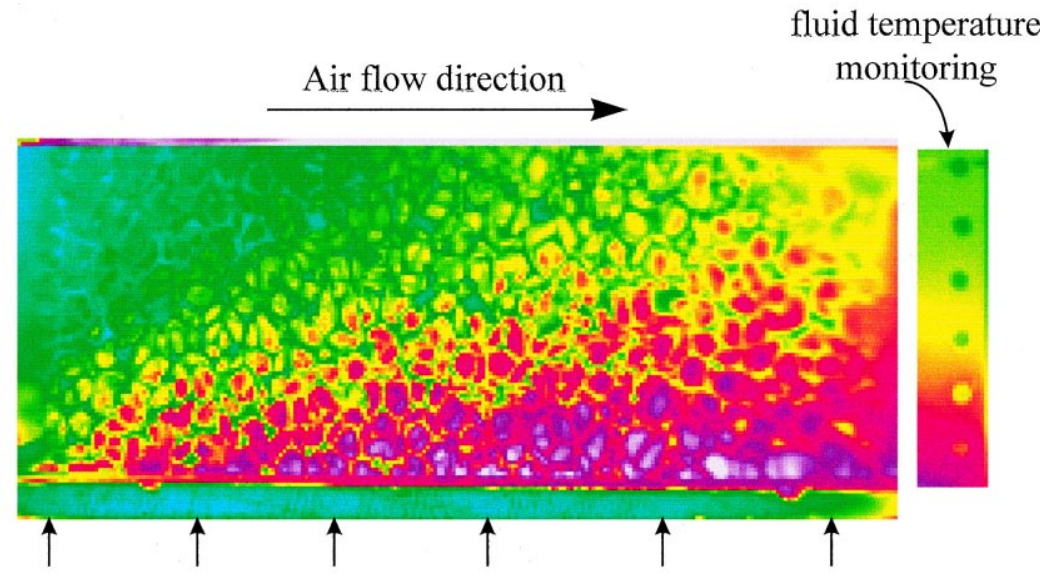

$\mathrm{mm}$

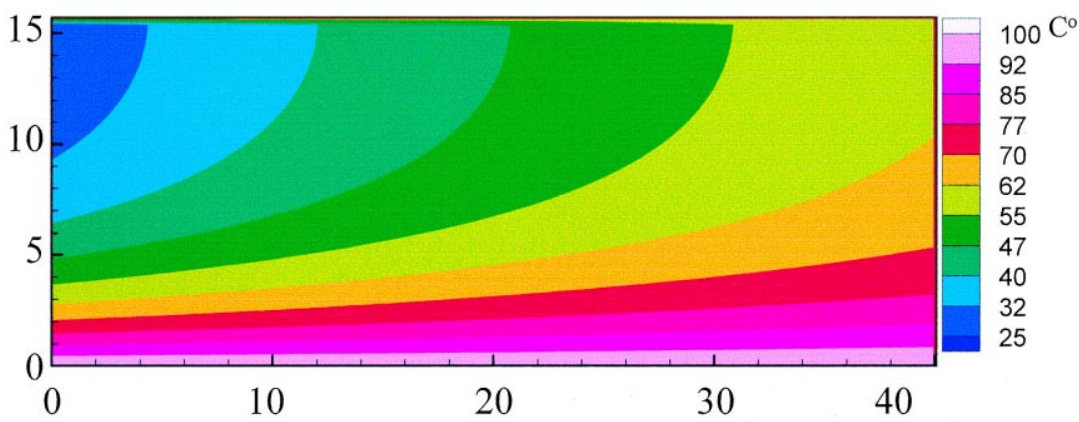

b)

(30PPI foam, $v=2 \mathrm{~m} / \mathrm{s}, \mathrm{b}=16 \mathrm{~mm}$.)

Fig. 11. (a) A thermal image of the $(x, z)$ plane taken of an open cell Al alloy foam in accordance with the cooling scheme shown on Fig. 2 [9]. (b) A simulation performed for a Biot number that most closely matches the experimental image [9]. 
(iv) Mapping of the simulations onto the measured temperatures establishes the coefficients.

(v) Subject to coincidence between simulations and measurements, the model is used to predict effects of the material variables on $H$ and the heat flux $Q$.

Heat transfer from the metal borders to the fluid, flowing at average velocity $v$, is considered to scale in accordance with the bank-of-cylinders model $[37,38]$ :

$$
B i=C\left(k_{\mathrm{f}} / k\right) \operatorname{Pr}^{0.36} \operatorname{Re}^{n}
$$

where $k_{\mathrm{f}}$ is the thermal conductivity of the fluid, $\operatorname{Pr}$ the Prandtl number, the Reynolds number $R e=v d / \gamma_{\mathrm{f}}$, with $\gamma_{\mathrm{f}}$ the kinematic viscosity, and $n$ is a coefficient dependent on the magnitude of $\operatorname{Re}$ (typically, $n=0.4$ ), the coefficient $C$ is about unity.

When the fluid temperature $T_{\mathrm{f}}(x)$, is assumed independent of $z$, the metal temperatures for the symmetric configuration shown in Fig. 2 are given by [14] $\left(T_{\mathrm{f}}<T\right)$ :

$$
T(z, x)=T_{\mathrm{f}}(x)+\left[T_{1}-T_{\mathrm{f}}(x)\right] \frac{\left[\sinh \left[2 \chi_{\mathrm{eff}}(D-z)\right]+\sinh \left(2 \chi_{\mathrm{eff}} z\right)\right]}{\sinh 2 \chi_{\mathrm{eff}} D}
$$

where $T(x, z)$ is the temperature of the metal, $T_{1}$ is the (constant) temperature of the heat source. The corresponding fluid temperature is [14]:

$$
T_{\mathrm{f}}(x)=\left(T_{1}-T_{0}\right) \exp (-x / l)
$$

where $T_{0}$ is the (constant) temperature of the incoming fluid and $l$ a transfer length. In the absence of a thermal resistance at the attachments, this length is:

$$
l=\frac{\rho_{\mathrm{f}} c_{\mathrm{p}} v D}{2 \eta k_{\mathrm{eff}} \sqrt{B i_{\mathrm{eff}}}}\left[1+\frac{\rho}{1.5 \eta} \tanh \left(\frac{2 D}{d} \sqrt{B i_{\text {eff }}}\right)\right]^{-1}
$$

where $c_{\mathrm{p}}$ is the specific heat of the fluid and $\eta=1-0.22 \rho$. Upon measuring the metal temperature $T(x, z)$, as well as the fluid temperature $T_{\mathrm{f}}(x), l$ can be determined such that the only unknown is $\chi_{\text {eff. }}$ If the metal temperatures can be adequately simulated by a single choice for $\chi_{\text {eff }}$, the model would be deemed adequate and predictions made.

The substrate attached to the cellular medium also contributes to the heat transfer. In the absence of a significant thermal constriction, this contribution may be added to $H_{\mathrm{c}}$, such that the overall heat transfer coefficient, $H_{0}$, is:

$$
H_{0} \approx H+\eta B i_{\mathrm{eff}} k_{\mathrm{eff}}
$$

More typically, there are interface effects that reduce $H_{0}$.

\subsection{Heat fluxes}

The heat $Q$ flowing into the fluid through the cellular medium per unit width is related to the heat transfer coefficient by: 


$$
Q=L H_{0} \Delta T_{\mathrm{lm}}
$$

where $L$ is the length of the medium (Fig. 2). Here $\Delta T_{\operatorname{lm}}$ is the logarithmic mean temperature. It is related to the temperature of the heat source $T_{1}$, as well as fluid temperature at the inlet, $T_{0}$ and that at the outlet, $T_{\mathrm{e}}$ by:

$$
\Delta T_{\operatorname{lm}}=\frac{T_{\mathrm{e}}-T_{0}}{\ln \left[\left(T_{1}-T_{0}\right) /\left(T_{1}-T_{\mathrm{e}}\right)\right]}
$$

Usually, $T_{1}$ and $T_{0}$ are specified by the application. Accordingly, $T_{\mathrm{e}}$ must be assessed from Eqs. (14) and (15) in order to determine $Q$.

\subsection{Thermal calibrations}

Experimentally, long ducts have been used subject to the fluid temperature everywhere being appreciably lower than the metal temperature [13]. Images obtained subject to these conditions (Fig. 11a) are mapped onto simulations based on Eqs. (13) and (14) for various $\chi_{\text {eff }}$ (Fig. 11b). Discrepancies arise because the fluid temperature is not independent of $z$, but the deviations appear to be small. The calibration indicates an effective thermal conductivity, $k_{\mathrm{eff}} \approx 0.28 k$, and an effective local heat transfer coefficient, $h_{\text {eff }} \approx 0.34 h[13]$.

Both effective values have coefficients smaller than unity because only about a third of the metal ligaments contribute to conduction and heat transfer.

\subsection{Pressure drop}

The pressure drop $\Delta p$ in the fluid along the cellular medium, length $L_{\mathrm{f}}$, is given by $[13,14,39]$ :

$$
\Delta p / L_{\mathrm{f}}=F \Omega_{\mathrm{g}} v^{2} / a
$$

where $F$ is a friction factor:

$$
F=\operatorname{Re}^{-\alpha} \phi_{\mathrm{P}}(\rho)
$$

The exponent $\alpha$ and the function $\phi_{\mathrm{P}}$ have been determined by experiment [13] such that $\alpha=0.4$ and $\phi_{\mathrm{P}}=4$. In practice, the pressure drop must be small enough to be compatible with the fans or pumps used to transmit the fluid through the medium, discussed next.

\subsection{Trade-off}

For any system there is a trade-off between heat flux and pressure drop. A cross plot of these two quantities in accordance with the non-dimensional parameters defined by the model (Table 1) illustrates this (Fig. 2). The solid lines indicate the trend between heat dissipation and pressure drop as the relative density increases, 
Table 1

Non-dimensional parameters governing the performance of cellular metal heat dissipation media. $a_{\mathrm{f}}=$ thermal diffusivity of cooling fluid

\begin{tabular}{ll}
\hline Heat flux & $\tilde{Q}=Q / k\left[T_{1}-T_{0}\right]$ \\
Prandtl number & $P r=v_{\mathrm{f}} / a_{\mathrm{f}}$ \\
Reynolds number & $\tilde{R} e=v L / v_{\mathrm{f}}$ \\
Cell wall thickness & $\tilde{d}=d / L$ \\
Foam thickness & $\tilde{D}=D / L$ \\
Nusselt number & $N u=B i k / k_{\mathrm{f}}$ \\
Thermal conduction & $\tilde{K}_{\mathrm{f}}=\sqrt{k_{\mathrm{f}} / k}$ \\
Power dissipation & $\tilde{p}=\Delta p D L^{2} / \rho_{\mathrm{f}} v_{\mathrm{f}}^{3}$ \\
\hline
\end{tabular}

at fixed cell size. The dotted lines indicate the effect of cell size at fixed density. Each cross-over point represents a specific cellular material.

The preferred material domain is indicated in Fig. 2. It is preferred because such materials achieve high heat dissipation at moderate pressure drop: that is, for cell size in the mesorange and with relative densities of order, $\rho \approx 0.2$. The particular material of choice can only be found by coupling Fig. 2 with the operating characteristics (back pressure and flow rate) of fans/blowers used to circulate the fluid. Such analysis has revealed that the trade-off between pressure drop and heat dissipation achievable with cellular metals is more propitious than that for conventional fin-pin arrays [2], enabling the design of substantially more compact heat sinks.

\section{Minimum weight structures}

\subsection{Structural indices}

Panels, shells and tubes subject to bending or compression have characteristics determined by structural indices [10,11,24,25,40-43]. These are obtained by deriving expressions for the stresses, displacements and weights in terms of the loads, dimensions, elastic properties and core densities. The details depend on the configuration, the loading and the potential failure modes, as elaborated below. There are a relatively small number of indices, based on weight, $\psi$, and load $\pi$. These can be expressed either in nondimensional form (Table 2) or in convenient dimensional forms. The associated material properties are summarized in Table 3 . For bending, it is convenient to define an additional structural index: the stiffness index, $S$. It is related to the elastic load index, $\pi_{\mathrm{e}}$, (Table 2 ) by:

$$
S=\Pi_{\mathrm{e}}(L / \delta)=(P / \delta) / B E_{\mathrm{s}}
$$

where $P$ is the load, $\delta$ the deflection, $L$ the span and $E_{\mathrm{s}}$ the Young's modulus for the material comprising the cellular medium. 
Table 2

Structural indices for foam core systems ${ }^{\mathrm{a}}$

\begin{tabular}{llll}
\hline Indices & Column & Panel & Shell \\
\hline Weight, $\psi$ & $W / \Omega L^{3}$ & $W / \Omega L^{2} B$ & $W / \Omega R^{2} L$ \\
Load (elastic), $\pi_{\mathrm{e}}$ & $P / E_{\mathrm{f}} L^{2}$ & $P / E_{\mathrm{f}} L B$ & $P / E_{\mathrm{f}} L R$ \\
Load (plastic), $\pi_{\mathrm{p}}$ & $P / \sigma_{y} L^{2}$ & $P / \sigma_{y} L B$ & $P / \sigma_{y} L B$ \\
\hline
\end{tabular}

anotation: $B=$ width; $c=$ core thickness; $d_{\mathrm{f}}=$ face thickness; $L=$ span length; $W=$ weight; $P=\operatorname{load} ; \alpha_{2}=\left(E / E_{\mathrm{s}}\right) \rho^{-2}$.

Table 3

Material properties

\begin{tabular}{llll}
\hline Property & Face & Foamed core & Solid unfoamed core \\
\hline Density $\left(\mathrm{kg} / \mathrm{m}^{3}\right)$ & $\Omega_{\mathrm{f}}$ & $\rho \Omega$ & $\Omega$ \\
Youngs's modulus $(\mathrm{GPa})$ & $E_{\mathrm{f}}$ & $E$ & $E_{\mathrm{s}}$ \\
Shear molulus & - & $G$ & - \\
Yield strength $(\mathrm{MPa})$ & $\sigma_{y}$ & $\sigma_{0}$ & $\sigma_{\mathrm{s}}$ \\
\hline
\end{tabular}

When optimizations are conducted simultaneously for weight and core density, explicit weight and deflection ratios result which, thereafter, greatly simplify determination of the relationships between the structural indices [1,43]. For example, stiffness-limited, laterally loaded panels containing a core with stiffness characterized by Eq. (20) exhibit minimum overall weight when the weight of the face sheets is a quarter of that for the core (Table 4). At this minimum, the contribution to the deflection by core shear is exactly twice that contributed by stretching the face sheet. Other weight ratios are summarized in Table 4.

Table 4

Relative core/face sheet weights at the minimum $[2,43]$

\begin{tabular}{llll}
\hline Configuration & Constraint & $\begin{array}{l}W_{\mathrm{c}} / W_{\mathrm{f}} \\
\text { Fixed } \rho\end{array}$ & Global \\
\hline Panel & Stiffness & 2 & 4 \\
& Strength & 1 & 4 \\
Tube & Stiffness & 1 & - \\
& Strength & $1 / 2$ & - \\
\hline
\end{tabular}


Table 5

Coefficients for laterally loaded panels [2]

\begin{tabular}{llcccc}
\hline Loading & & $A_{1}$ & $A_{2}$ & $A_{3}$ & $A_{4}$ \\
\hline Cantilever & End & 3 & 1 & 1 & 1 \\
\multirow{3}{*}{ Three point bending } & Uniform & 8 & 2 & 2 & 1 \\
\multirow{2}{*}{ Ends build in } & Central & 48 & 4 & 4 & 2 \\
& Uniform & $384 / 5$ & 8 & 9 & 2 \\
& Central & 192 & 4 & 9 & 2 \\
& Uniform & 384 & 8 & 12 & 2 \\
\hline
\end{tabular}

\subsection{Stiffness-limited sandwich structures}

Panels that experience lateral loads are often stiffness limited. Stiffness also affects the natural vibration frequencies. That is, high stiffness at low weight increases the resonant frequencies, thereby facilitating their avoidance.

Choosing minimum weight configurations is relatively straightforward whenever the design loads allow choices entirely within the elastic range. The basic concepts can be found in several literature sources. The key results are reiterated to establish the procedures, as well as to capture the most useful results. For all bending problems, a series of non-dimensional coefficients, designated $A_{i}$, relate the deflections to the moments. These have been comprehensively summarized elsewhere $[1,2,50]$. They are repeated for convenience (Table 5). They will be used throughout the following deviations.

\subsubsection{Method of analysis}

An analysis based on sandwich beams is first used to demonstrate the procedure. The compliance in bending is:

$$
\begin{aligned}
S^{-1}= & \left(2 / A_{1}\right)\left(L / d_{\mathrm{f}}\right)(L / c)^{2} \\
& +\left(1 / A_{2}\right)(L / c)(E / G)
\end{aligned}
$$

The first term is the contribution from face sheet stretching and the second from core shear. The corresponding weight index is:

$$
\psi=\frac{2 d_{\mathrm{f}}}{L}+\rho \frac{c}{L}
$$

The free variables subject to optimization are $d_{\mathrm{f}}, c$ and $\rho$.

If the relative core density $\rho$ is fixed, the optimization proceeds in accordance with the following five steps:

(i) Express Eq. (21) in terms of the face thickness, $d_{\mathrm{f}}$.

(ii) Substitute $d_{\mathrm{f}}$ into the weight function (22).

(iii) Minimize Eq. (22) with respect to the core thickness: $\mathrm{d} W / \mathrm{d} c=0$, to obtain the optimum core thickness, $c_{*}$. 
(iv) Specify the compliance, $\delta / P$, and span length, $L$. Substitute $c_{*}$ into Eq. (21) to obtain the optimum face thickness, $d_{\mathrm{f}}^{*}$.

(v) The minimum weight is determined by inserting $d_{\mathrm{f}}^{*}$ and $c^{*}$ into Eq. (21).

The key results of such an optimization have been summarized in Table 6 and Fig. 12a.

\subsubsection{Global minimum}

A more complete optimization is possible if the density of the core is also treated as a free variable. Paradoxically, in this case, the results are analytical, because the displacements contributed by core shear are twice those from bending and the core weight is four times that of the face sheets (Table 4). These ratios give the relationship:

$$
S=\left(A_{1} / 60\right)(c / L)^{2} \Psi
$$

Here it is assumed that the core and face sheets are made from the same materials (so that $\Omega=\Omega_{\mathrm{f}}$ and $E_{\mathrm{s}}=E_{\mathrm{f}}$ ).

At the weight minimum, the core thickness is explicitly related to the stiffness by:

$$
\frac{c}{L}=2\left[\frac{18 \alpha_{2} A_{2} S}{A_{1}^{2}}\right]^{1 / 5}
$$

Substituting $c / L$ into Eq. (23) gives the interrelationship:

$$
\Psi=\frac{15 S^{3 / 5}}{A_{1}^{1 / 5}\left(18 \alpha_{2} A_{2}\right)^{2 / 5}}
$$

For plotting purposes, it is convenient to re-express Eq. (25) in the form

$$
Y=3.19 X^{3 / 5}
$$

where $Y=\Psi \sqrt{8 A_{1} / 3 \alpha_{2} A_{2}}$ and $X=S \sqrt{A_{1}} /\left(3 \alpha_{2} A_{2} / 8\right)^{3 / 2}$. This result has also been plotted in Fig. 12a.

Table 6

Formulae for laterally loaded, stiffness-limited panels and beams ${ }^{\mathrm{a}}$

\begin{tabular}{lllll}
\hline Condition & $\begin{array}{l}\text { Core thickness } \\
\left(c^{*} / L\right)\end{array}$ & $\begin{array}{l}\text { Minimum weight } \\
(Y)\end{array}$ & $\begin{array}{l}\text { Core relative density } \\
(\rho)\end{array}$ & $\begin{array}{l}\text { Face thickness } \\
\left(d_{\mathrm{f}}^{*} / L\right)\end{array}$ \\
\hline Global minimum & $2\left[\frac{18 \alpha_{2} A_{2} S}{A_{1}^{2}}\right]^{1 / 5}$ & $3.19 X^{3 / 5}$ & $0.59 X^{2 / 5}$ & $\frac{A_{1}}{96 \alpha_{2} A_{2}}\left(\frac{c}{L}\right)^{3}$ \\
Fixed $\rho^{\mathrm{b}}$ & $\frac{4\left(d_{\mathrm{f}} / L\right)}{\rho(1-2 \xi)}$ & $2 \sqrt{2} \rho^{3 / 2} \sqrt{\xi}\left[\frac{3-2 \xi}{1-2 \xi}\right]$ & - & {$\left[\frac{3 \alpha_{2} A_{2}}{4 A_{1}} \xi R^{3}\right]^{1 / 4}$} \\
\hline
\end{tabular}

$\quad \Omega=\Omega_{\mathrm{f}}, E_{\mathrm{s}}=E_{\mathrm{f}}$.
${ }^{\mathrm{b}} \xi$ is related to the weight index $X$ by: $X=\frac{16 \sqrt{2} \rho^{5 / 2} \xi^{3 / 2}}{1-4 \xi^{2}} . Y=\psi \sqrt{8 A_{1} / 3 \alpha_{2} A_{2}}, X=S \sqrt{A_{1}} / 3\left(3 \alpha_{2} A_{2} / 8\right)^{3 / 2}$. 


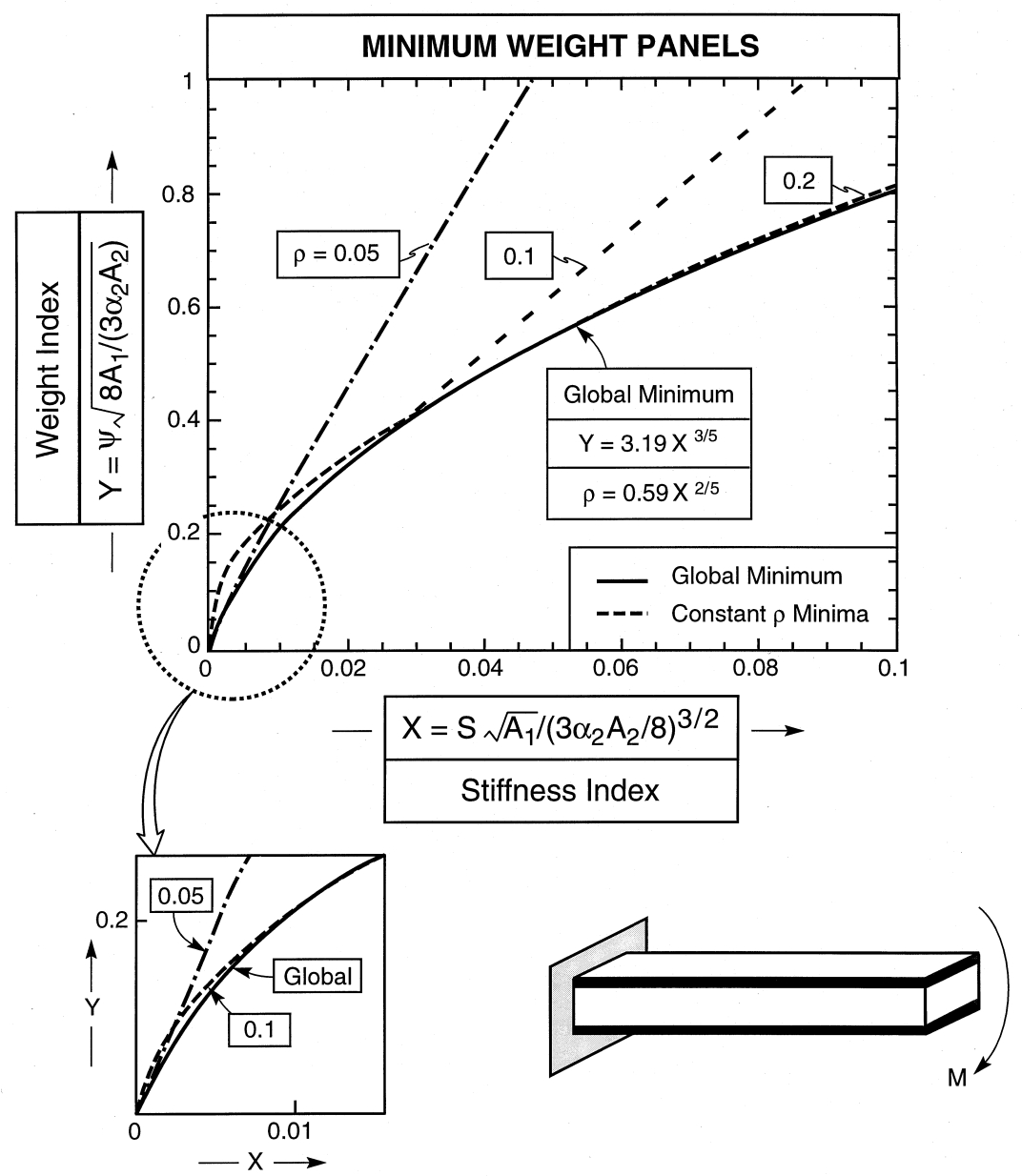

Fig. 12. Minimum weight analysis for stiffness-limited, laterally loaded panels. (a) A cross plot of the minimum weight and stiffness indices showing the global minimum, as well as minima for three fixed densities ( $\rho=0.05,0.1$ and 0.2 ). Note that, for the global minimum, the core density is given by $\rho=0.59 X^{2 / 5}$. (b) A schematic illustrating the domains wherein yielding of either the face sheets or the core prohibit use of elastic analysis. A more comprehensive analysis is needed to establish minimum weights in these domains. The location of the domain boundaries is affected by the load indices $Z_{\mathrm{f}}$ and $Z_{\text {c }}$. These must be specified by the application before using the diagram. For this choice of load index $\left.\pi_{\mathrm{p}}=10^{-4}\right)$ and yield strain $\left(\epsilon_{\mathrm{Y}}=\epsilon_{\mathrm{s}}=0.007\right)$, core yielding will arise in minimum weight panels designed at stiffness below $A^{\prime}$. The corresponding points for face yielding is $A$.

For each stiffness, there is a corresponding relative density for the core:

$$
\rho=0.59 X^{2 / 5}
$$

There is also an explicit face thickness, 


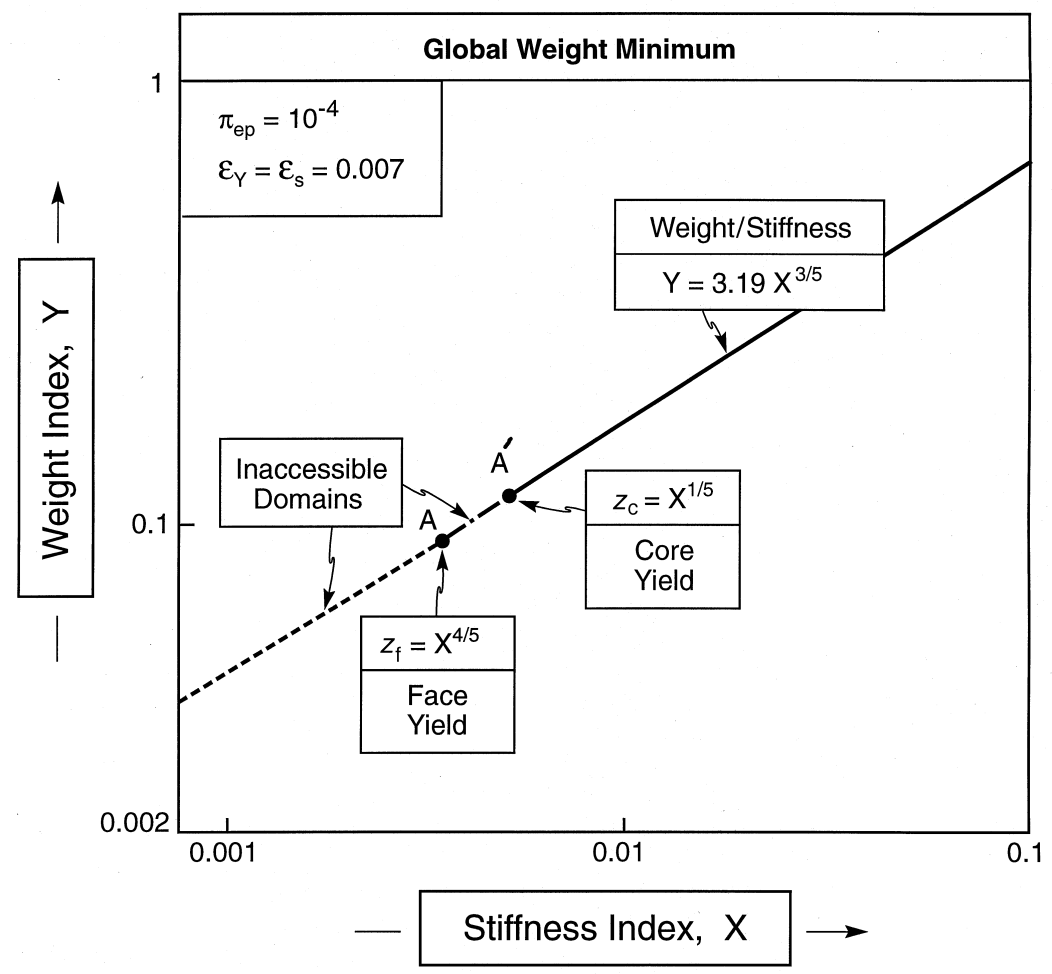

Fig. 12(b). Continued.

$$
\frac{d_{\mathrm{f}}}{L}=\frac{A_{1}}{96 \alpha_{2} A_{2}}\left(\frac{c}{L}\right)
$$

\subsubsection{Failure limits}

Application of these weight diagrams is limited by the occurrence of yielding, either of the face sheets or in the core, and by face wrinkling [1,11]. Face yielding commences when the maximum tensile or compressive stress caused by bending reaches the tensile yield strength, $\sigma_{\mathrm{Y}}$. For the globally optimal sandwich, this result can be expressed in terms of $X$ :

$$
Z_{\mathrm{f}} \geq X^{4 / 5}
$$

where

$$
Z_{\mathrm{f}}=\left[\frac{16 A_{1}}{48^{4 / 5} \alpha_{2} A_{2} A_{3}}\right] \Pi_{\mathrm{p}}
$$


For a given transverse load, $P$, all globally optimized designs less stiff than that associated with the equality in Eq. (29) exceed yield in the face sheets. The behavior is illustrated on Fig. 12b, for one choice of $\pi_{\mathrm{p}}$, where the equality of Eq. (29) is represented by a point, $A$. That is, configurations having lower stiffness than $A$ cannot be realized at the weights given by Eq. (29). Weights in excess of the global minimum would be needed to obtain stiffnesses in this range. Note that logarithmic axes have been used (rather than linear, Fig. 12a) to highlight the inadmissible range.

For the optimally designed sandwich, core yielding occurs when:

$$
Z_{\mathrm{C}} \geq X^{1 / 5}
$$

where

$$
Z_{\mathrm{C}}=\left(\frac{P}{B L \tau_{\mathrm{c}}}\right) \sqrt{\frac{A_{1}}{\alpha_{2} A_{2}}}\left[\frac{1}{48^{1 / 5} A_{4}}\right]
$$

with a similar interpretation to that for face yielding, indicated in Fig. $12 \mathrm{~b}$ as point $A^{\prime}$. Again lower stiffnesses cannot be realized at the global weight minimum, this time because of core yielding.

Accordingly, structures that realize global weight minima are those requiring high stiffness. This happens because the face sheet thicknesses need to achieve minimum weights increase substantially as the stiffness index increases (relative to core thickness and density). At lower stiffnesses, because of the thinner face sheets (28) and lower core densities (27) yielding is likely to intervene. For yielding to be avoided, the weight must be increased above the minimum by increasing either the face sheet thickness or the core density.

\subsection{Stiffened panels}

Competition for sandwich systems is comprised principally of waffle-stiffened panels (Fig. 13). For comparison, it is convenient to re-express Eq. (25) in the form:

$$
\frac{W}{B L^{2}}=\Omega\left(\frac{P / \delta}{B E_{\mathrm{f}}}\right)^{3 / 5}\left[\frac{15}{A_{1}^{1 / 5}\left(18 \alpha_{2} A_{2}\right)^{2 / 5}}\right]
$$

For a waffle panel subject to bending about one of the stiffener directions, the weight and stiffness are related by:

$$
\frac{W}{B L^{2}}=\frac{72}{5} \Omega_{0}\left(\frac{P / \delta}{E_{\mathrm{o}} A_{1} B}\right)\left(\frac{L}{d_{\mathrm{s}}}\right)^{2}
$$

where the dimension $d_{\mathrm{s}}$ is defined in Fig. 13, with $E_{\mathrm{o}}$ Young's modulus for the material comprising the panel and $\Omega_{\mathrm{o}}$ its density. 


\section{COMPETING CONCEPTS}

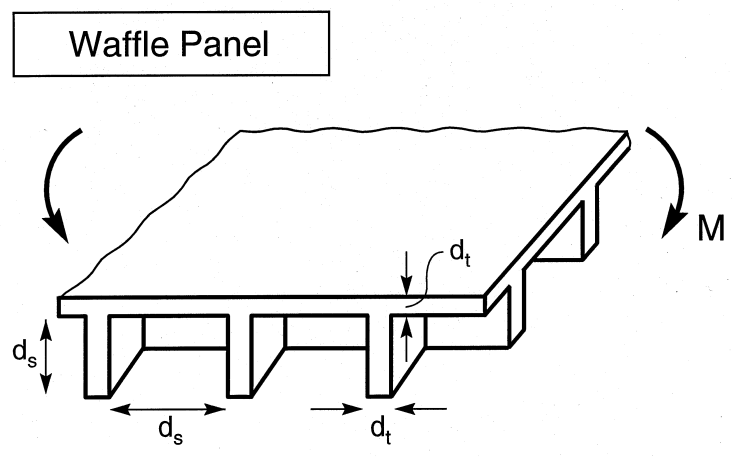

\section{Sandwich Panel}

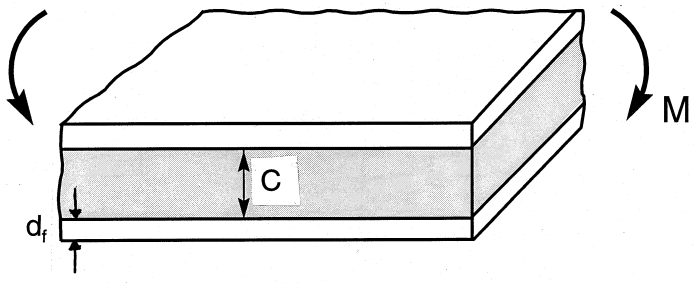

\section{SIMILAR STIFFNESS AT EQUIVALENT DEPTH}

Fig. 13. A schematic comparing a waffle-stiffened panel with a sandwich panel both loaded in bending.

Equating the weights of the sandwich and waffle panels, Eqs. (31) and (32) give:

$$
\frac{d_{\mathrm{s}}}{L}=\sqrt{\frac{24}{25}\left(\frac{\Omega_{\mathrm{o}}}{\Omega}\right)\left(\frac{E_{\mathrm{f}}}{E_{\mathrm{o}}}\right)}\left(\frac{P / \delta}{B E_{\mathrm{f}}}\right)^{1 / 5}\left(\frac{18 \alpha_{2} A_{2}}{A_{1}^{2}}\right)^{1 / 5}
$$

Comparison of (33) with the optimized sandwich panel (Eqs. (20) and (24)) yields, at equivalent weight:

$$
\frac{d_{\mathrm{s}}}{c}=\frac{\sqrt{6}}{5} \sqrt{\left(\frac{\Omega_{\mathrm{o}}}{\Omega}\right)\left(\frac{E_{\mathrm{f}}}{E_{\mathrm{o}}}\right)}
$$

This result is stiffness independent because Eqs. (33) and (24) have the identical 
functional dependence. Accordingly, a waffle panel made from the same material as a sandwich panel $\left(\Omega=\Omega_{\mathrm{o}}, E_{\mathrm{f}}=E_{\mathrm{o}}\right)$ has a slightly smaller overall thickness, at the same weight and stiffness. The choice, therefore, depends entirely on manufacturing cost and durability.

\subsection{Cylindrical shells}

Strength-limited sandwich structures can be weight competitive with stiffener reinforced designs: the lowest weight designs in current usage [44,45]. Shells are a more likely candidate for sandwich construction than axially compressed panels or columns. This preference arises because both hoop and axial stresses are involved, enabling the isotropy of sandwich panels to be exploited. There are two basic requirements for sandwich shells: (i) sufficient core shear stiffness is needed for adequate buckling strength; and (ii) the shear yield strength of the metal foam must be large enough to maintain the buckling resistance of the shell, particularly in the presence of imperfections. Numerical methods are needed to determine minimum weights of both sandwich and reinforced systems. Simple analytical estimates are not possible. Some prototypical results are presented to illustrate the configurations wherein sandwich construction may be preferred.

One configuration comprises cylindrical sheets subject to axial loads (Fig. 14), optimized with respect to $d_{\mathrm{f}}$ and $c$, subject to prescribed core density. They regard the fully dense core material as identical to the face sheet material $\left(\Omega_{\mathrm{f}}=\Omega_{\mathrm{s}}\right.$, and $E_{\mathrm{f}}=E_{\mathrm{s}}$ ), and use a core with stiffness at the low end of the range found for commercial materials. The face sheets are elastic-perfectly plastic with compressive yield strength $\sigma_{\mathrm{Y}}$. Note that, at the optimum weight, and in the range where the face sheets experience yield, the compressive stress in the face sheets associated with elastic buckling is coincident with the yield strength in compression, $\sigma_{\mathrm{Y}}$. The weight index has been determined at a representative yield strain for Al alloys $\left(\epsilon_{\mathrm{Y}}=0.007\right)$. These results are independent of the length of the cylinder. The buckling mode is axisymmetric.

For comparison the structural performance has been calculated for an optimally designed, axially stiffened cylindrical shell with hat-shaped stiffeners located on the inside. These results apply to a shell segment located between rings spaced a distance $L$ apart, with $L / R_{\mathrm{s}}=1$. A lower $L / R_{\mathrm{s}}$ would have a lower weight index, and vice versa. Note that, over the range plotted, the shell buckles elastically for the chosen yield strain. (Shells with stiffeners on the outside of the cylinder have somewhat greater buckling strength and, thus, a lower weight index, but outside stiffening is often excluded for other reasons.) This example illustrates that metal foam core sandwich shells can have a competitive advantage over established structural methods of stiffening, particularly at relatively low structural indices.

To pursue the subject further, the sandwich shells are optimized with respect to relative core density $\rho$, as well as $d_{\mathrm{f}}$ and $c$. Simultaneously, the consequence of 

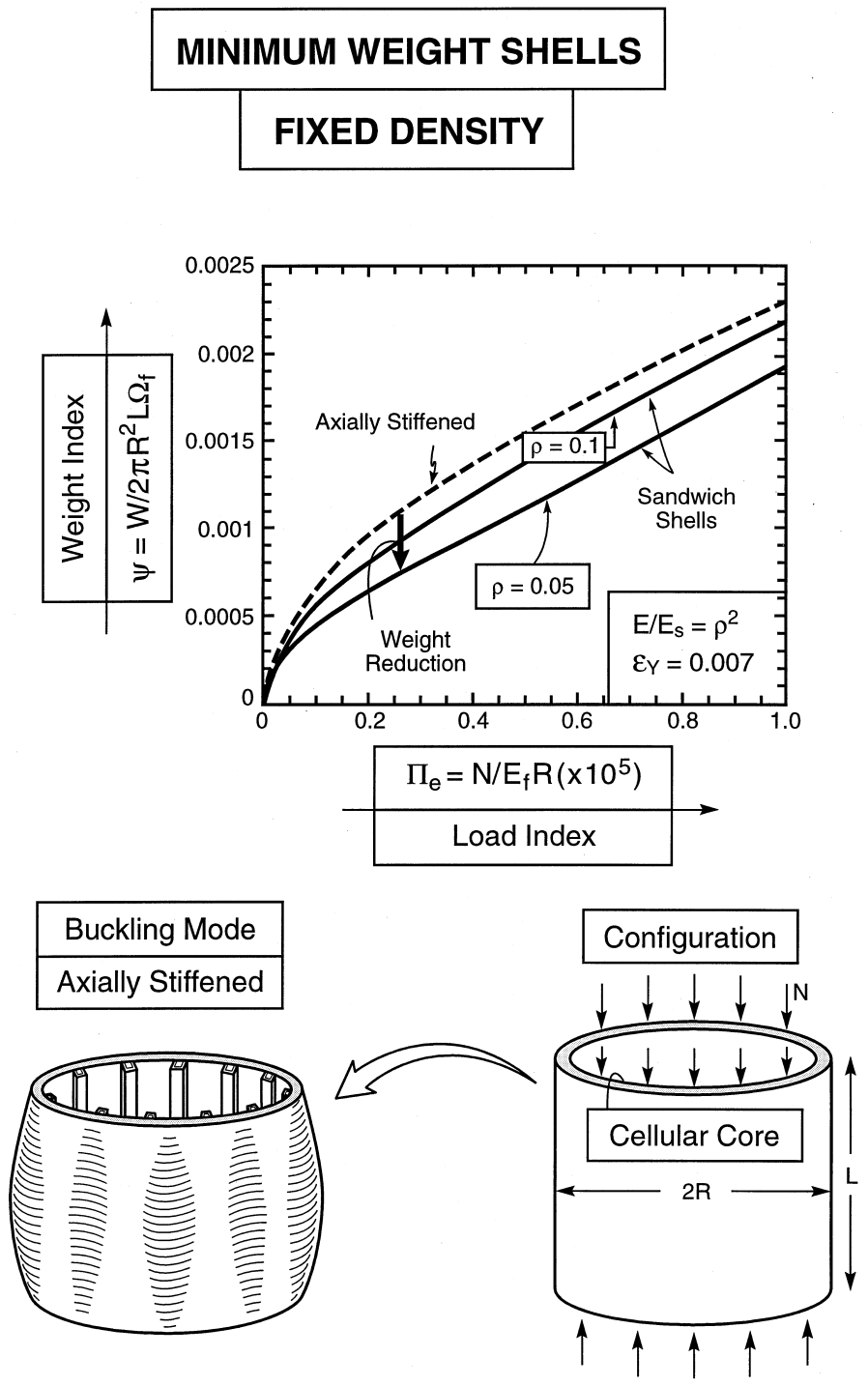

Fig. 14. Minimum weight comparisons for strength-limited, axially compressed cylindrical sandwich shells at fixed density (with $\alpha_{2}=1$ ) having dimension $L / R_{\mathrm{s}}=1$, compared with those for a shell with inside stiffeners.

using a core with superior stiffness is addressed by assuming a core having properties comparable to the best commercial materials. The results for the fully optimized foam core sandwich shells are plotted in Fig. 15 with accompanying plots for the optimal relative density of the core. For reference, the result for the optimally designed cylindrical shell with axial hat-stiffeners is repeated from Fig. 14. This comparison illustrates both the weight superiority of foam metal 


\section{MINIMUM WEIGHT SHELLS}

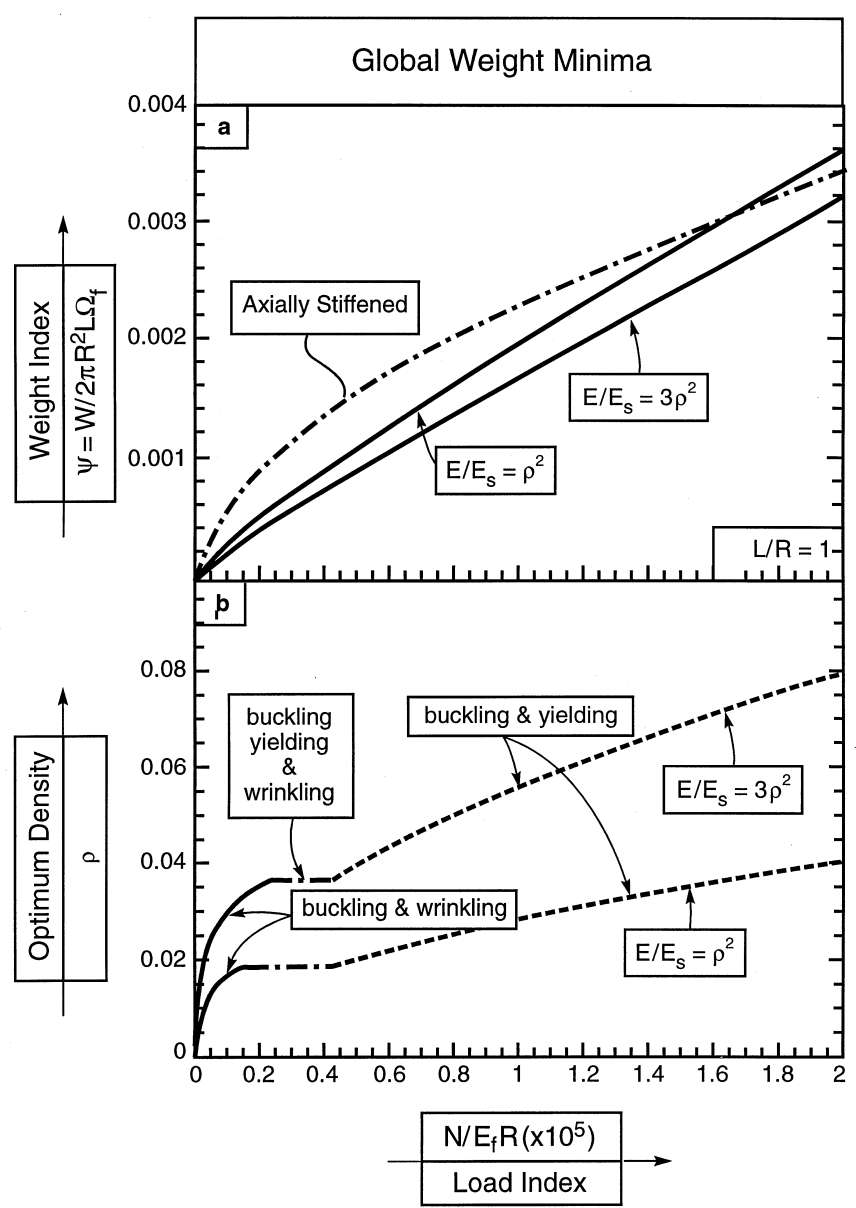

Fig. 15. (a) Minimum weights of sandwich construction for two core stiffness behaviors compared with those for stiffened shells. Here the minimization has been conducted on both weight and core density. The stiffnesses were chosen to encompass those found experimentally $\left(1 \geq \alpha_{2}, \geq 4\right.$, Fig. 6). (b) The relative densities at the weight minimum represented by (a).

core sandwich shells over conventional shell construction and the potential benefit to be gained by using a core material with the best available stiffness. At the lowest core densities, face wrinkling is expected to become the weight limiting failure mechanism (see Fig. 18). While analysis of this mechanism would be needed to establish specific weight benefits, optimized sandwich construction is still expected to afford lower weights than hat-stiffening. 


\section{Minimum Weight Axially Compressed Panels}

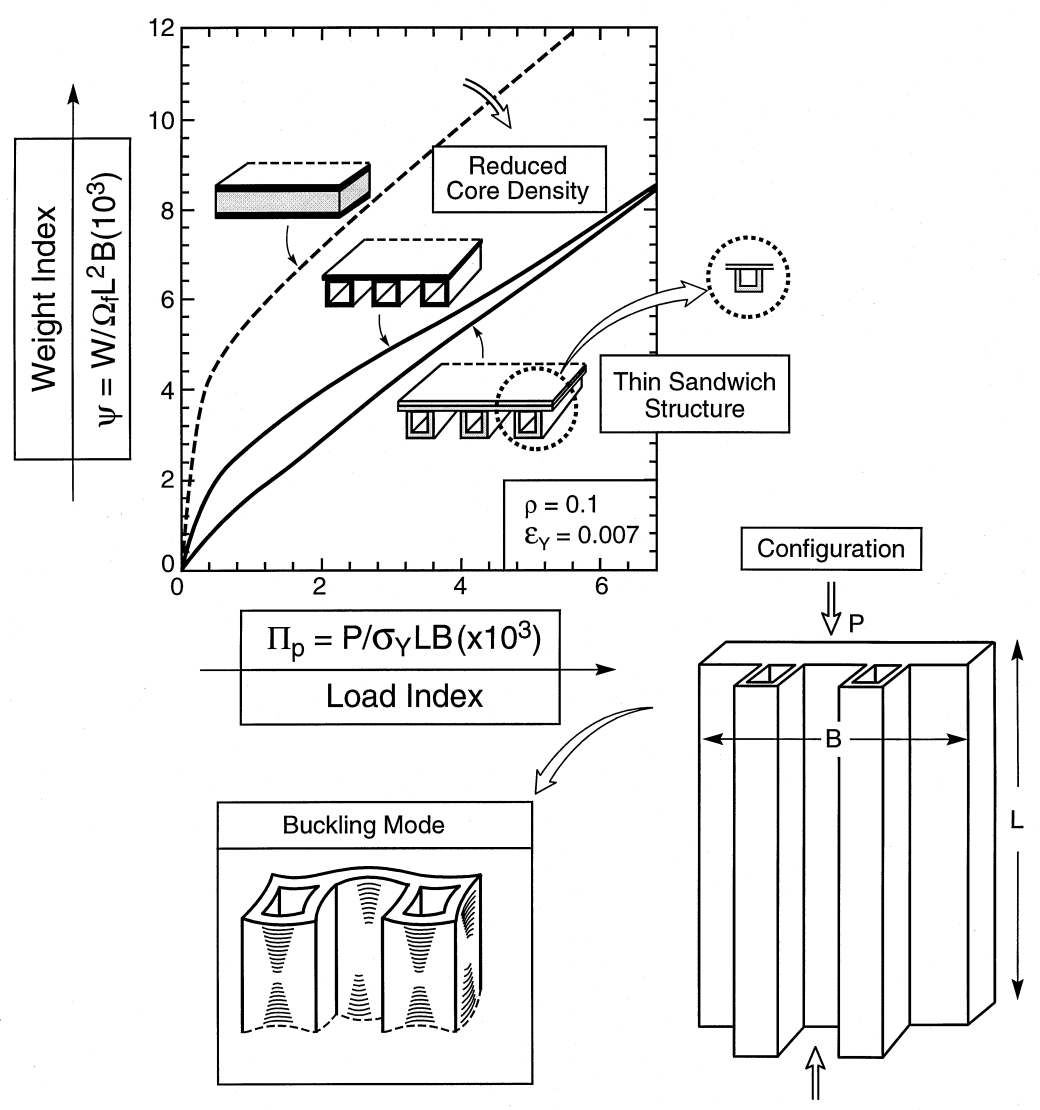

Fig. 16. Minimum weight calculations for strength-limited, axially compressed panels with fixed core density $(\rho=0.1)$.

\subsection{Imperfection sensitivity}

An important consideration for strength-limited thin walled construction concerns the influence of imperfections. In most cases, imperfections reduce the buckling loads, sometimes considerably. In shells, imperfections cause out-of-plane bending, which lowers the maximum support load due to two effects: (i) by advancing nonlinear collapse; and (ii) by causing premature plastic yielding, which reduces the local stiffness of the shell and, in turn, hastens collapse. Since they 
always exist, practical designs take this imperfection sensitivity into account. Generally, experimental results establish a knockdown factor on the theoretical loads that may be used as the design limit with relative impunity. The influence of imperfections can also be addressed by numerical simulations.

\section{GLOBAL WEIGHT MINIMA}
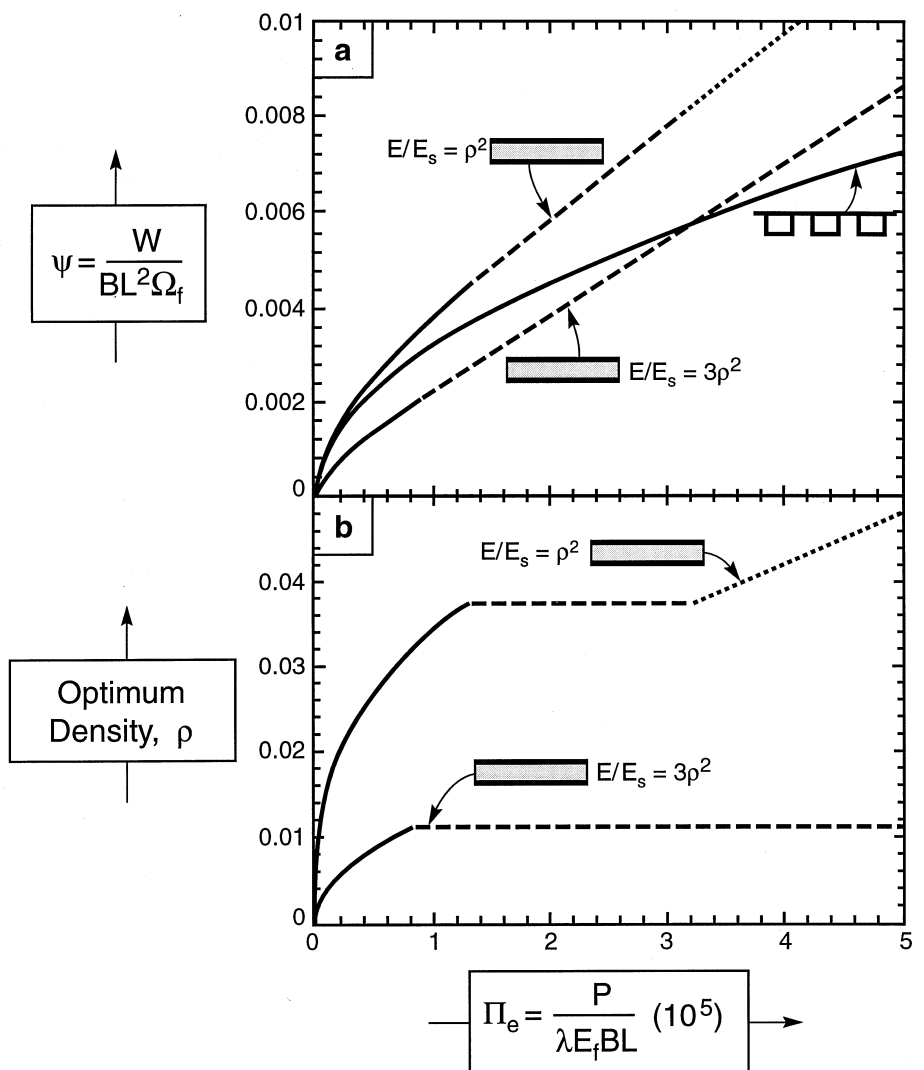

wrinkling, buckling

$$
\begin{array}{cc}
\begin{array}{c}
\text { wrinkling, yielding, } \\
\text { buckling }
\end{array} & \begin{array}{l}
\text { yielding, } \\
\text { buckling }
\end{array}
\end{array}
$$

Fig. 17. (a) Minimum weight results for strength-limited, axially compressed sandwich panels with variable core density. The operative failure modes change as the load index changes, as indicated. (b) The core densities corresponding to (a). Here $\lambda$ in the abscissa is the knockdown factors due to imperfections. 


\subsection{Other configurations}

Corresponding diagrams for panels and columns are presented in Fig. 16-18 [11]. The associated buckling modes are indicated in the insets. Results for minimum weight sandwich panels at a fixed cored density, $\rho=0.1$ (Fig. 16) are not especially promising. There is only a small domain of weight savings. This domain arises when sandwich construction is used within the stringers, as well as the panels, of a stringer-stiffened configuration. This construction has lowest weight at small levels of load index.

\section{AXIALLY COMPRESSED COLUMNS}

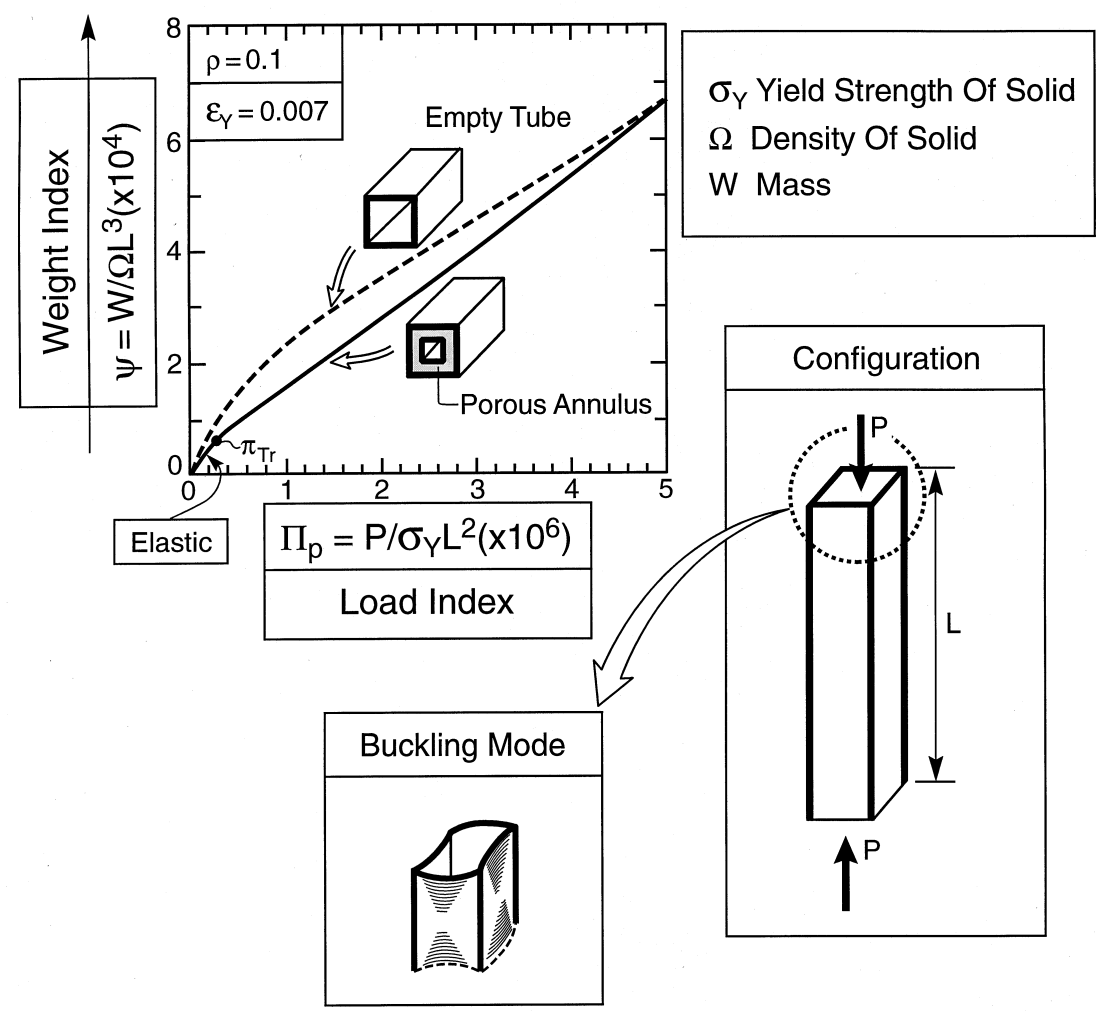

Fig. 18. Minimum weight calculations for axially compressed strength limited square tubes with fixed core density $(\rho=0.1)$. 
Further minimization with core density leads to more pronounced weight savings (Fig. 17). In this case, even flat sandwich panels can have lower weight than stringer-stiffened panels, especially at lower levels of load index. Note that the failure modes governing the weight change as the load index changes and that the minimum weights coincide with simultaneous occurrences of either two or three modes. The challenge in taking advantage of the potential weight savings arises in manufacturing and relates to the low relative densities required to realize these performance levels (Fig. 17). Moreover, these low $\rho$ need to be achieved while maintaining acceptable morphological quality.
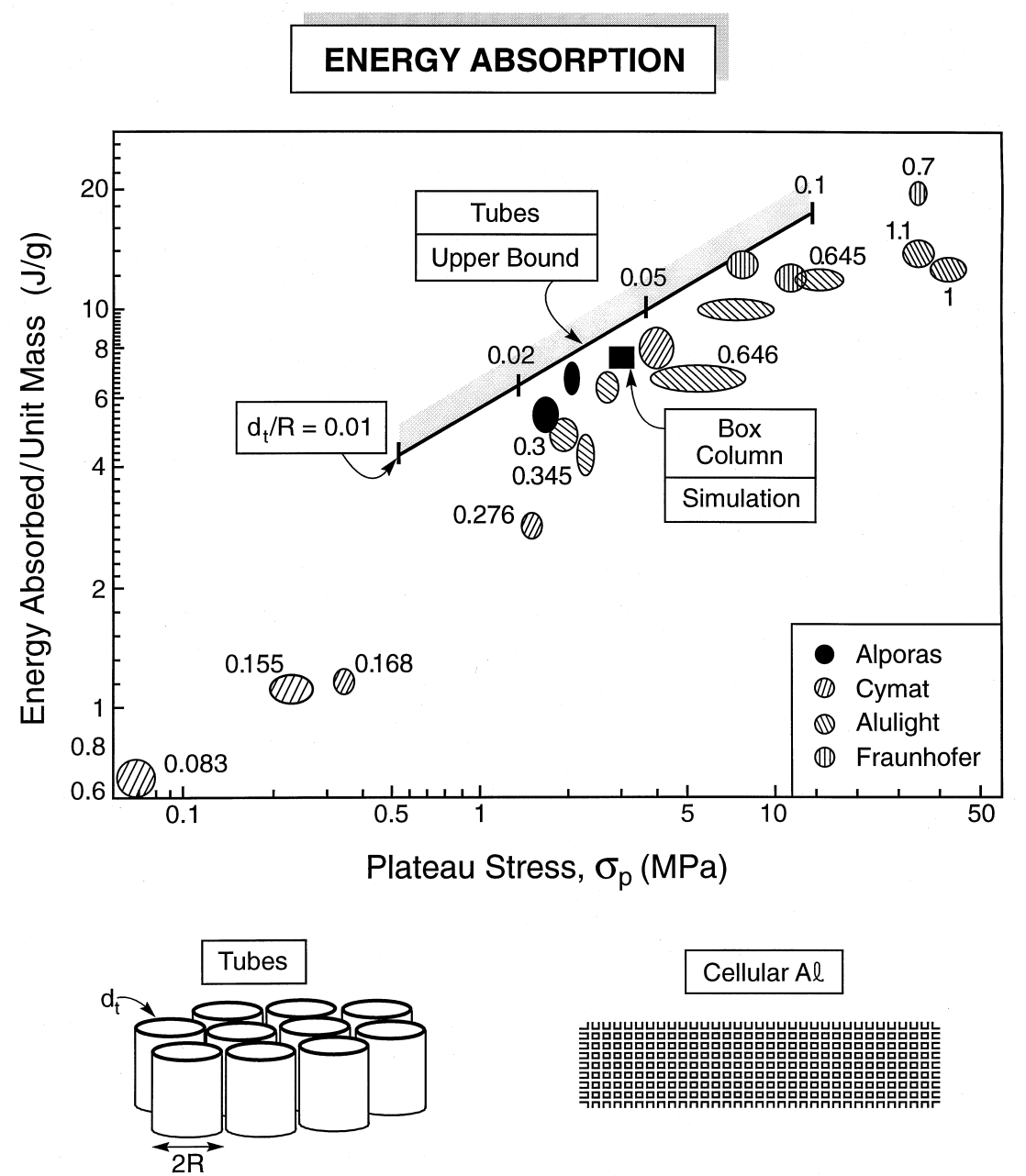

Fig. 19. Energy per unit mass against plateau stress for cellular Al alloys. Also shown is a comparison of the energy absorption per unit mass for Al tubes. 
Results for columns (Fig. 18) indicate that thin walled sandwich tubes are lighter than foam-filled and conventional tubes, but the beneficial load ranges are small.

\subsection{Synopsis}

For those wishing to explore cellular metal core sandwich construction, the following recommendations are made based on the above considerations.

(i) Determine the constraints that govern the structure and, in particular, whether it is stiffness or strength controlled.

(ii) If stiffness is dominant, there is a relatively straightforward procedure for determining minimum weights. This entails using the formulae summarized in the tables. It is important to realize that there will always be lighter configurations (especially optimized honeycomb or waffle panels). Those configurations should be explicitly identified, whereupon a manufacturing cost and durability comparison can be made that determines the viability of sandwich construction. Other qualities of the cellular metal may bias the choice. Moreover, it is important to calculate the domains wherein the weights based on elasticity considerations cannot be realized, because of the incidence of 'inelastic' modes: face yielding, core yielding, face wrinkling. Some help in assessing these limits has been provided. Future additions to the Manual [2] will provide more general results.

(iii) When strength (particularly buckling) controls the design, the rules governing sandwich construction are less well formulated. In general, numerical methods are needed to compare and contrast this type of construction with stiffened systems. Some general guidelines are given in the Manual [2]; these facilitate deliberations about loadings and configurations more likely to benefit from sandwich construction. Configurations unlikely to benefit are also described. It is recommended that, where benefits seem likely, detailed simulations and testing should be used to assess the viability of sandwich construction.

\section{Energy absorption}

\subsection{Nondimensional indices}

A comprehensive treatment of energy absorption by foams is given in Gibson and Ashby [1]. A few salient results are repeated here for completeness. The densification strain, $\epsilon_{\mathrm{d}}$ (Fig. 1), is dictated solely by the relative density, such that $[1,43]$ :

$$
\epsilon_{\mathrm{d}}=1-\rho \phi_{v}
$$

where $\phi_{\mathrm{v}}$ is a measure of the relative void space retained when the cells have 
collapsed. It is about 1.4. The energy absorption per unit volume, $U$, is:

$$
U / \sigma_{\mathrm{p}}=\epsilon_{\mathrm{d}}
$$

Accordingly, a plot of $U$ against $\sigma_{\mathrm{p}}$ is dictated by the densification strain, causing all data for cellular $\mathrm{Al}$ alloys to reside along a diagonal band. The corresponding result for the energy absorbed per unit mass, $U_{\mathrm{p}}$, is:

$$
\begin{aligned}
U_{\mathrm{p}} / \sigma_{\mathrm{p}} & =\epsilon_{\mathrm{d}} / \rho \Omega \\
& \equiv \frac{\left(1-\rho \phi_{\mathrm{v}}\right)}{\rho \Omega}
\end{aligned}
$$

A plot $U_{\mathrm{p}}$ against $\sigma_{\mathrm{p}}$ now has an additional dependence on density, but since $\sigma_{\mathrm{p}}$ also depends on density, Eq. (3), the data for cellular Al alloys still reside within a relatively narrow band (Fig. 19), with less than a factor 2 spread in energy absorption among the better quality commercial materials. Specifically, upon using Eq. (3) as representative of $\sigma_{\mathrm{p}}$ for closed cell materials, the realizable energy absorption becomes

$$
U_{\mathrm{p}} / \sigma_{\mathrm{s}}=\alpha_{3}\left(1-\rho \phi_{\mathrm{v}}\right) / \Omega
$$

The energy absorption can only be appreciably increased by elevating the plateau stress. Accordingly, when the allowable stress is specified by the application, the energy is largely predetermined by the inherent deformation characteristics of the material. The only significant materials issue concerns the ability to adjust the stress in order to attain higher energy absorption. Higher levels of energy absorption per unit mass could be accessed by eliminating the knockdown factor on yield strength and achieving a more uniform plateau stress, but only at correspondingly larger stress. Accordingly, for energy absorption purposes, there is minimal motivation for manufacturing developments that enhance the morphological quality beyond that achievable in the better commercially available materials.

\subsection{System comparison}

Competition for cellular materials is provided by a bank of thin-walled columns. In columnar configurations, the energy is absorbed through plastic buckling of the walls. The collapse of tubes and their energy absorption have been analyzed as follows. The force needed for axial crushing is $[2,46,47]$ :

$$
P_{\mathrm{c}}=4 \pi R_{\mathrm{s}}^{1 / 3} d_{\mathrm{t}}^{5 / 3} \sigma_{\mathrm{s}}
$$

where $d_{\mathrm{t}}$ is the tube thickness. The associated crushing stress, $\sigma_{\mathrm{p}}$, is:

$$
\begin{aligned}
\sigma_{\mathrm{p}} & \equiv P_{\mathrm{c}} / \pi R_{\mathrm{s}}^{2} \\
& =2^{1 / 3}\left(2 d_{\mathrm{t}} / R_{\mathrm{s}}\right)^{5 / 3} \sigma_{\mathrm{s}}
\end{aligned}
$$

but the relative density of the tube is 


$$
\rho=2 d_{\mathrm{t}} / R_{\mathrm{s}}
$$

such that the crushing stress becomes

$$
\sigma_{\mathrm{p}}=2^{1 / 3} \rho^{5 / 3} \sigma_{\mathrm{s}}
$$

The densification strain for a tube is:

$$
\begin{aligned}
\epsilon_{\mathrm{d}} & \equiv\left(1-2 d_{\mathrm{t}} / R_{\mathrm{s}}\right) \\
& =(1-\rho)
\end{aligned}
$$

The energy absorbed per unit volume is thus:

$$
\begin{aligned}
U / \sigma_{\mathrm{s}} & \equiv \sigma_{\mathrm{p}} \epsilon_{\mathrm{d}} \\
& =2^{1 / 2} \rho^{5 / 3}(1-\rho)
\end{aligned}
$$

The corresponding energy per unit mass is

$$
U_{\rho} / \sigma_{\mathrm{s}}=2^{1 / 2} \rho^{2 / 3}(1-\rho) / \Omega
$$

Since Al tubes can be made with yield strength $\sigma_{\mathrm{s}} \approx 200 \mathrm{MPa}$, these energy absorptions can be superposed onto the cellular Al data for comparison purposes (Fig. 19). The comparison suggests that Al alloy tubes are superior to cellular alloys on a weight basis. However, two additional considerations enable the cellular materials to be competitive in some cases:

(i) Numerical simulations of column crushing [12] indicate stress oscillations as plastic buckling progresses, resulting in energy levels about two-thirds those expected from Eq. (45).

(ii) Tubes absorb efficiently only upon axial loading. They are much less effective when impacted obliquely. Cellular media are isotropic and omnidirectional. Accordingly, when impacts from a range of directions are expected, foams are attractive.

\subsection{Impact}

Upon impact, kinetic energy $\kappa\left(\equiv m v^{2} / 2\right)$ from the object must be dissipated by plastic work. The impact can be fully absorbed, without exceeding the stress $\sigma_{\mathrm{p}}$, if the foam thickness, $D$, satisfies:

$$
D \geq \kappa / U A_{\mathrm{I}}
$$

where $A_{\mathrm{I}}$ is the area over which the impact is spread by interaction with the buffer plate. The minimum weight of cellular material, $W_{\min }$, needed to absorb the impact is:

$$
W_{\min }=\kappa / U_{\rho}
$$


Hence, the energy absorption metric $U_{\rho}$ in Eq. (29) (Fig. 19), characterizes the minimum weight.

\subsection{Blast amelioration}

Blast from explosions results in an impulse being imparted to the structure. Momentum from the impulse, I, per unit area, is acquired by the buffer plate, which then compresses the cellular material [2,33]. The requirement is that the resulting kinetic energy in the buffer be fully dissipated within the foam, while the stress on the structure never exceeds that at the plastic front. Since cellular metals have rate-insensitive yield strengths at the strain-rates relevant to typical blasts $[2,33,48]$, this stress is still the quasistatic value, $\sigma_{\mathrm{p}}$. The foam thickness needed to absorb the blast is thus:

$$
D \geq I^{2} / 2 \Omega_{\mathrm{b}} d_{\mathrm{b}} U
$$

where $\Omega_{\mathrm{b}}$ is the density of the buffer plate and $d_{\mathrm{b}}$ its thickness. Consequently, the overall minimum weight is:

$$
W *_{\min } / W_{\mathrm{b}}=1+\left(I / d_{\mathrm{b}}\right)^{2} / 2 \Omega_{\mathrm{b}} U_{\rho}
$$

where $W_{\mathrm{b}}$ is the weight of the buffer. Again, $U_{\rho}$ is the important metric for the foam.

Blast amelioration applications for cellular metals would appear to be favored by their isotropy, relative to honeycombs and tubes, as well as their high energy absorption relative to polymers [16]. Some appreciation can be gained by means of the following case study [2]. The impulse from an explosion decreases with distance, $x$, in the manner depicted in Fig. 20. Except for distances close to the explosion, $I$ varies in approximate accordance with:

$$
I \approx B_{I} M^{1 / 3} \exp \left[-C_{*}\left(x / M^{1 / 3}\right)\right]
$$

where $M$ is the mass of explosive and the coefficients are: $B_{I}=5 \times 10^{3} \mathrm{sPa}(\mathrm{kg})^{-1 / 3}$, $C *=1.3(\mathrm{~kg})^{-1 / 3} / \mathrm{m}$. Inserting $I$ into Eq. (48) relates the foam thickness to the parameters for the explosion $(M, x)$. A map characterizing the overall behavior is presented in Fig. 21, for a material having a plateau flow stress of $1 \mathrm{MPa}$ [33]. The map shows the thickness and weight of material needed to protect against explosions. For example, for a $50 \mathrm{~kg}$ explosion located $3 \mathrm{~m}$ from a structure that can sustain a pressure of $1 \mathrm{MPa}(10 \mathrm{~atm})$, the form should be $0.5 \mathrm{~m}$ thick and (with the buffer) weigh $200 \mathrm{~kg} / \mathrm{m}^{2}$. This is much more effective than the protection that could be obtained with polymer foams, tubes, etc. 


\section{BLAST PROTECTION}
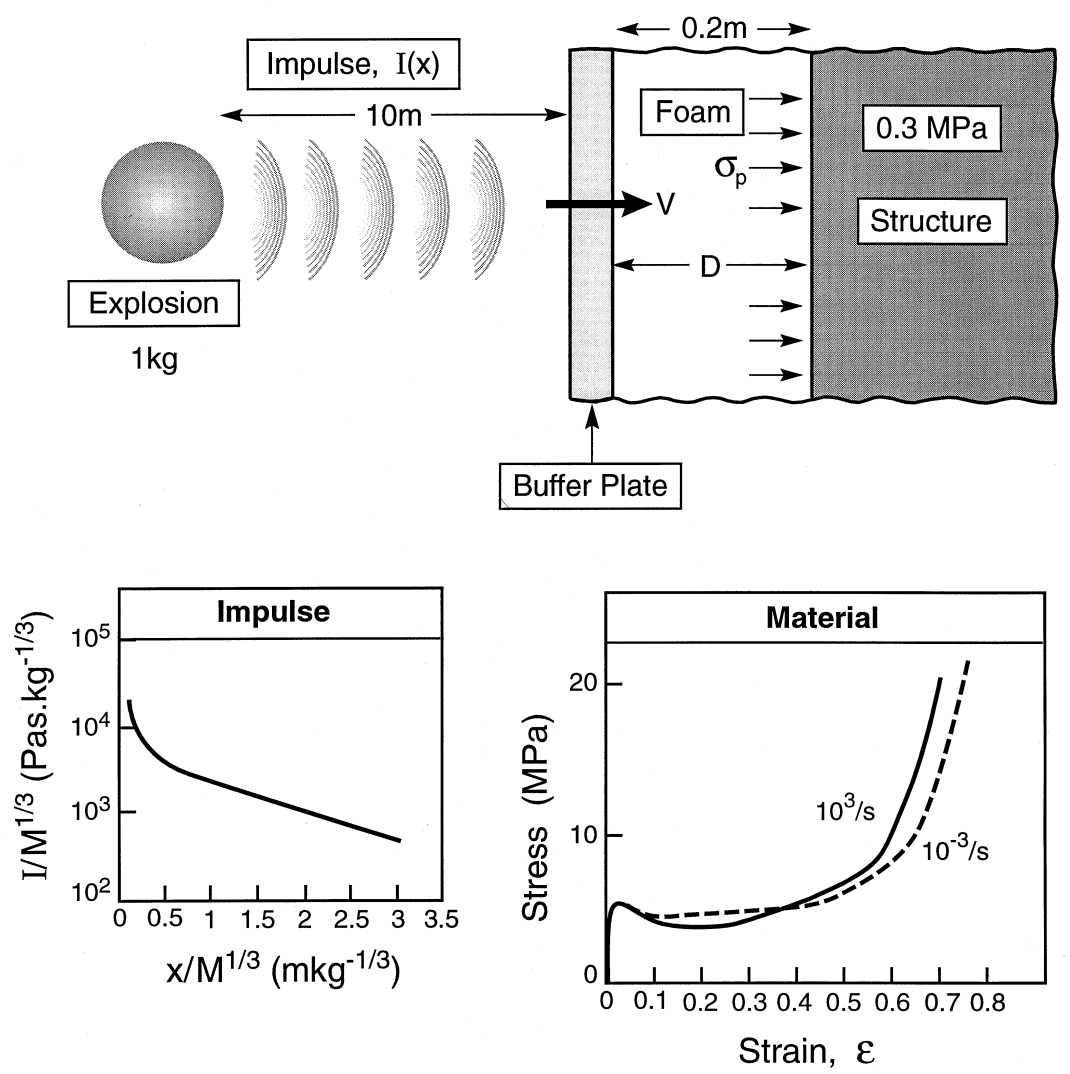

Fig. 20. The case study characterizing the blast amelioration behavior of $\mathrm{Al}$ alloy foams.

\section{Manufacturing status}

One of the challenges established by the above analysis is the need to create closed cell metals for ultralight sandwich structures with mechanical properties approaching the best achievable (Eqs. (1) and (3)). An additional capability for integral bonding with face sheets is implied. Beyond current capabilities, there is also a need for materials with much lower density cores $(\rho \ll 0.1)$ that, nevertheless, have the best achievable properties. A status report on two basic manufacturing categories potentially capable of realizing these possibilities is examined in this section. These are gas expansion and hollow sphere methods. 


\begin{tabular}{|c|c|}
\hline \multicolumn{2}{|c|}{ BLAST PROTECTION } \\
\hline $10 \mathrm{~atm}$ Structure & $\sigma_{\mathrm{p}}=1 \mathrm{MPa}$ \\
\hline
\end{tabular}

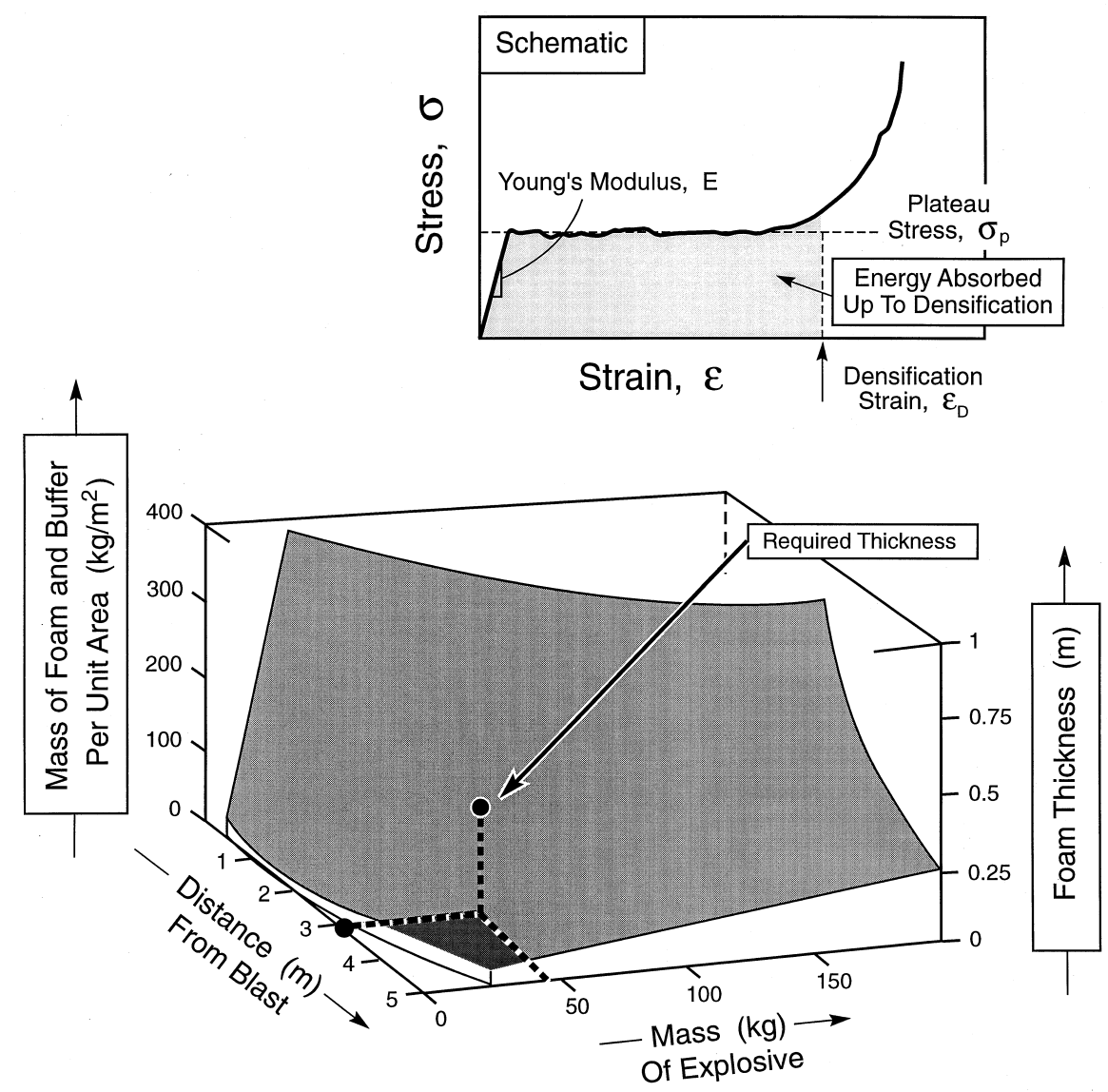

Fig. 21. A blast absorption surface for an Al foam with a plateau flow of $1 \mathrm{MPa}$. The example indicates the thickness of foam needed to absorb the blast from a $50 \mathrm{~kg}$ explosion located $3 \mathrm{~m}$ away from a structure capable of sustaining a $10 \mathrm{~atm}$ pressure.

\subsection{Gas expansion methods}

Foam casting and powder metallurgy approaches predominate among the gas expansion methods. Typically, an internal gas pressure is used to expand a porous metal with closed porosity. In several cases, the expansion is conducted within a dense shell that becomes the integrally bonded face layer. The gas pressure is 
derived by one of three methods: (i) it is released from a dispersed particulate, such as $\mathrm{H}_{2}$ from $\mathrm{TiH}_{2}$ [3,4]; (ii) it derives from high pressure generated within an entrapped inert gas [6,7]; or (iii) a gas is injected into a liquid metal [5]. Method (i) has been applied to Al alloys and, very recently, to steels. Method (ii) has been used with Ti alloys. Method (iii) has been implemented for particulate reinforced Al alloys (PRA).

One embodiment of method (i) mixes Al powders with hydride particles and cold consolidates into ribbons (e.g. by extrusion) [4]. Segments of the ribbon are emplaced within a thin-walled container that becomes the dense structural constituent. Upon heating above the solidus, when the gas is released, foaming produces a closed cell porous core bonded to the container. In its present manifestation, the method creates an isotropic, cellular core, but with occasional large voids. Sandwich panels and foam filled tubular configurations have been made. The method has unparalleled versatility for creating foam filled shapes. This attribute has particular merit for applications insensitive to the mechanical properties of the cellular material. It has not yet been possible to conduct the foaming process with the control needed to contemplate ultralight structures requiring core properties that approach the best achievable levels.

A second embodiment of (i) used for Al alloys mixes the hydride powder into the molten alloy within a container, to cause foaming [3]. Additions of Ca to the alloy enable a relatively high viscosity to be realized at temperatures in the twophase (liquid/solid) domain, just above the solidus. A hydrogen overpressure is used to control cell development within the liquid. Large blocks are produced by this method with relatively uniform, isotropic cells. This material has the best relative properties (highest $\alpha_{1}$ in Eq. (1) and $\alpha_{3}$ in Eq. (3)) among presently available commercial Al alloys [20,21]. This uniformity is enabled by the high viscosity achieved in the liquid and the hydrogen overpressure used during foaming. This embodiment has not yet been used to produce material with integrally bonded face sheets. Such structures have been produced by cutting sheets from the as-manufactured blocks, by electrodischarge machining (EDM) and either polymer bonding or brazing to face sheets [49]. This manufacturing approach offers the best structural performance in applications, such as ultralight shells, where weight savings require superior core properties.

Method (ii) is primarily of interest for $\mathrm{Ti}$ alloys [6,7]. It is based on hot isostatic pressing (HIPing), with several atmospheres of Ar introduced into the can containing the alloy powder. The Ar is trapped at high pressure within residual voids upon HIPing and subsequent rolling/forming. A final heat treatment causes the voids to expand, resulting in a sandwich structure. The process is limited to a core containing $30-40 \%$ porosity. This limitation prohibits the weight savings elaborated in Section 5. However, the material competes in applications when contoured or curved thin sheets are now used, without stiffeners, for reasons of fabricability or cost. Blistering between the core and the face sheets is a present impediment to manufacturing by this method.

In method (iii), high pressure air injected into the molten PRA through orifices causes foaming and allows for a continuous process that generates sheets of 
porous material $\approx 10 \mathrm{~cm}$ thick [5]. The material tends to be anisotropic with large cells, subject to a relatively frequent incidence of defects [20,21]. However, the lower manufacturing costs represent an important attribute, especially for applications insensitive to the mechanical properties, such as energy absorption.

\subsection{Hollow sphere methods}

Thin walled hollow spheres of various alloys can be produced by two intrinsically different methods and then consolidated into a structure. One method uses slurries [8] and the other involves gas atomization [9]. Thus far, hollow spheres of $\mathrm{Ti}$ and $\mathrm{Ni}$ alloys, as well as steels, have been produced. The approach is inherently attractive, because being solid state, the process is amenable to a stringent control regimen. It thus has the potential to realize the properties expressed by Fig. 5. Such realizations would enable those ultralight structures that demand superior core properties.

(i) Slurries of metal oxide or hydride powders are blown into thin walled spheres and sintered, employing a commercial process now used for ceramic spheres [8]. They are subsequently converted into the metal by annealing at the requisite gas phase partial pressures, such as a low oxygen pressure for steel spheres made from iron oxides.

(ii) When alloy powders are made by atomization, Ar can be trapped in the liquid droplet. It then expands, as the drop projects, until it solidifies, creating hollow powders. The hollow fraction can be separated by flotation [9].

Consolidation of the hollow spheres can be achieved either by isostatic pressing or by liquid phase sintering. This aspect of the manufacturing has yet to receive systematic attention. It is crucially important, since the properties are so sensitive to the area fraction achieved at each of the sphere contacts (Fig. 5).

\section{Summary}

The connections between the morphological quality of cellular metals and the requirements for their implementation comprise: (i) those insensitive to the thermomechanical properties of the material; and (ii) others that are strongly influenced by cellular material quality. This distinction partitions the connection between manufacturing and implementation.

(a) Several applications categories are insensitive to morphological quality, provided that some reasonable minimum is consistently achieved. These comprise energy absorption applications and some ultralight panels and tubes. The latter category includes some stiffness limited structures, as well as strength limited configurations subject to low imperfection sensitivity. 
(b) Other applications require that the cellular materials have the best achievable thermomechanical properties. One category comprises imperfection sensitive ultralight shells and circular tubes that operate in the elastic range. In such cases relatively high strength cores, approaching the best achievable, are essential to the realization of substantial weight savings. Another category comprises open cell heat dissipation media.

Within these overall material property benchmarks, comparisons with competing materials and systems suggest the following three implementation opportunities:

(i) For heat dissipation purposes, cellular metals are unique. Moreover, there are substantial opportunities to greatly improve their thermal performance by tailoring cell size and density. The manufacturing challenge is demanding, but justified by the performance benefit.

(ii) Cellular $\mathrm{Al}$ alloys are attractive in those applications that require exceptional energy absorption, yet are compatible with moderately high stress delivery levels (1-10 MPa). Manufacturing requirements are not especially stringent for these applications, enabling use of lower cost process methods. The isotropy of the foams and the uniformity of their force delivery represent performance advantages over competing concepts, such as thin walled box columns.

(iii) Strength and stiffness limited ultralight structures designed within the elastic range all exhibit a domain wherein weight benefits arise from the use of thin sandwich construction comprising cellular metal cores. A subset of these structures have sufficient performance benefit to justify implementation. Others may have utility because of lower manufacturing cost. Preliminary attempts at defining structures that provide weight savings have identified panels and shells as opportunities. The greatest benefits appear to arise with relatively long strength-limited shells subject to axial compression. There also appear to be opportunities for stiffness limited panels that experience lateral loads. There are no benefits for compression structures designed with a load index in the plastic range. The requirements on the mechanical properties of the cellular material are themselves subject to the imperfection sensitivity of the structure. For imperfection insensitive structures, the dictates on properties are minimal, but the benefits from using a cellular core are also small. Conversely, imperfection sensitive structures, such as cylindrical shells, benefit most from having cellular cores with properties approaching the best achievable levels, with no knockdown. Cellular metal sandwich construction would provide even greater weight benefit if the density of the core could be substantially decreased below presently available materials $(\rho \ll 0.1)$, subject to mechanical properties that approach best-achievable levels (Eqs. (1) and (3)). Attainment of such materials constitutes a longer range manufacturing objective. 


\section{Acknowledgements}

The authors wish to thank the Defense Advanced Research Projects Agency and the Office of Naval Research for their sponsorship. Many individuals and groups have contributed to its contents. They include Professor B. Budiansky, Professor H. Stone, Dr R. Miller, Dr A. Bastawros, Dr Y. Sugimura and Hilary Bart-Smith of the Division of Engineering and Applied Sciences, Harvard University; Professor L. J. Gibson of the Department of Materials, MIT; Professor N. A. Fleck, Dr Y. J. Lu, Dr Anne-Marie Harte, Dr Angela Murphy, Dr V. Deshpande, K. McCullough and J. B. Olurin of the Micromechanics Center, Engineering Department, Cambridge University.

\section{Appendix A}

\section{A.1. Appendix $A$}

\section{A.1.1. Minimum weight transversely loaded panels with specified stiffness}

The stiffness, $s$, of a transversely loaded sandwich panel can be written as $[1,43,50]$ :

$$
\frac{1}{S} \equiv \frac{B \delta}{P}=\frac{2 L^{3}}{A_{1} E_{\mathrm{f}} d_{\mathrm{f}} d_{\mathrm{c}}^{2}}+\frac{L}{A_{2} d_{\mathrm{c}} G}
$$

where the dimensionless coefficients $A_{1}$ and $A_{2}$ depend only on the specific loading and support condition (Table 5). The first term in Eq. (A1) is the contribution from bending while the second is due to shearing of the core. It has been assumed that $d_{\mathrm{c}} \gg d_{\mathrm{f}}$, and that the core itself makes negligible contribution to the bending stiffness. The weight of the panel is

$$
W / B L=\Omega_{\mathrm{f}}\left(2 d_{\mathrm{f}}+\rho d_{\mathrm{c}}\right)
$$

assuming the face sheet density is that of the fully dense core $\left(\Omega_{\mathrm{f}}=\Omega\right)$.

Two optimization problems are considered for minimizing weight for a specified stiffness, or, equivalently, for maximizing stiffness at a specified weight. In the first, the global optimum is obtained by maximizing $s$ with respect to $d_{\mathrm{f}}, d_{\mathrm{c}}$ and $\rho$, subject to specified $W$. In the second, the relative core density $\rho$ is specified and thus removed as a variable in the maximization process. The core shear modulus is considered to vary with core density in accordance with Eq. (2), such that, with $v=1 / 3$,

$$
G / E_{\mathrm{s}}=(3 / 8) \alpha_{2} \rho^{2}
$$


The auxillary function used in the optimization process is

$$
f\left(d_{\mathrm{f}}, d_{\mathrm{c}}, \rho\right)=\frac{2 L^{3}}{A_{1} d_{\mathrm{f}} d_{\mathrm{c}}^{2}}+\frac{8 L}{3 A_{2} d_{\mathrm{c}} \alpha_{2} \rho^{2}}+\lambda\left(2 d_{\mathrm{f}}+\rho d_{\mathrm{c}}\right)
$$

where $\lambda$ is a Lagrangian multiplier introduced to enforce the constraint of specified weight. Because the sum of the first two terms in Eq. (A4) are proportional to the inverse of the stiffness, minimizing $f\left(d_{\mathrm{f}}, d_{\mathrm{c}}, \rho\right)$ maximizes $s$.

The outcome of the global minimization is as follows. The weight of the core is exactly four times the combined weight of the two face sheets, and the contribution to $1 / s$ in Eq. (A1) from the second term due to core shear is exactly twice that of the contribution from the first term due to the bending stiffness $[1,43]$. The first implies

$$
W / L B=10 d_{\mathrm{f}} \Omega_{\mathrm{f}}=(5 / 4) d_{\mathrm{c}} \rho \Omega_{\mathrm{f}}
$$

while the latter result gives

$$
\frac{1}{s}=\frac{6 L^{3}}{A_{1} E_{\mathrm{f}} d_{\mathrm{f}} d_{\mathrm{c}}^{2}}
$$

There are also specific values of $d_{\mathrm{c}}, d_{\mathrm{f}}$ and $\rho$ at the stiffness maximum given by [1],

$$
\begin{aligned}
& \frac{d_{\mathrm{c}}}{L}=2\left[\frac{18 \alpha_{2} A_{2} s}{A_{1}^{2} E_{\mathrm{f}}}\right]^{1 / 5} \\
& \frac{d_{\mathrm{f}}}{L}=\frac{A_{1}}{96 \alpha_{2} A_{2}}\left(\frac{d_{\mathrm{c}}}{L}\right)^{3}=\frac{A_{1}}{12 \alpha_{2} A_{2}}\left[\frac{18 \alpha_{2} A_{2} s}{A_{1}^{2} E_{\mathrm{f}}}\right]^{3 / 5} \\
& \rho=8 \frac{d_{\mathrm{f}}}{d_{\mathrm{c}}}=\frac{A_{1}}{3 \alpha_{2} A_{2}}\left[\frac{18 \alpha_{2} A_{2} s}{A_{1}^{2} E_{\mathrm{f}}}\right]^{2 / 5}
\end{aligned}
$$

In terms of the stiffness and weight variables,

$$
X=\frac{A_{1}^{1 / 2}}{\left(3 \alpha_{2} A_{2} / 8\right)^{3 / 2}} \frac{s}{E_{f}} \quad \text { and } \quad Y=\left(\frac{A_{1}}{3 \alpha_{2} A_{2} / 8}\right)^{1 / 2} \frac{W}{\Omega_{\mathrm{f}} B L^{2}}
$$

the global optimum satisfies

$$
Y=\frac{5}{16}(48 X)^{3 / 5} \text { with } \rho \frac{1}{8}(48 X)^{2 / 5}
$$

The minimization of $f\left(d_{\mathrm{f}}, d_{\mathrm{c}}, \rho\right)$ with respect to $d_{\mathrm{f}}$ and $d_{\mathrm{c}}$ with $\rho$ specified leads to the following parametric results for $X$ and $Y$

$$
X=\frac{16 \sqrt{2} \rho^{5 / 2} \xi^{3 / 2}}{1-4 \xi^{2}} \text { and } Y=2 \sqrt{2} \rho^{3 / 2} \xi^{1 / 2} \frac{(3-2 \xi)}{(1-2 \xi)}
$$


where

$$
\xi=\frac{A_{1}}{\left(3 \alpha_{2} A_{2} \rho^{3} / 4\right)}\left(\frac{d_{\mathrm{f}}}{L}\right)^{2}
$$

Each value of $\xi$ generates a minimum weight panel for the prescribed stiffness and the prescribed core density. The associated core thickness can be found from

$$
\frac{d_{\mathrm{c}}}{d_{\mathrm{f}}}=\frac{4}{\rho(1-2 \xi)}
$$

\section{References}

[1] Gibson LJ, Ashby MF. Cellular Solids, 2nd ed.. Cambridge: Cambridge University Press, 1997.

[2] Ashby MF, Evans AG, Hutchinson JW. Cellular Metals, a Design Guide. Cambridge: Cambridge University, Engineering Department, 1998.

[3] Akiyama S, Imagawa K, Kitahara A, Nagata S, Morimoto K, Nishikawa T, Itoh M. Foamed metal and method for producing same. US Patent no. 4,712,277, 1987.

[4] Baumeister J, Schrader J. Methods for manufacturing foamable metal bodies. German Patent no. DE 4,101,630, 1991.

[5] Jin I, Kenny L, Sang H. Method of producing lightweight foamed metal. US Patent no. 4,973,358, 1990.

[6] Martin RL, Lederich BJ. Powder Metallurgy Conference and Exhibition, Chicago, IL. In: Advances in Powder Metallurgy. Princeton, NJ: Metal Powder Industries Federation, 1991. p. 361-370.

[7] Kearns MW, Blenkinsop PA, Barber AC, Farthing TW. Int J Powd Met 1988;24:59.

[8] Nagel AR, Hurysz KM, Lee KJ, Cochran JK, Sanders TH Jr. Closed cell steel foams: fabrication and mechanical testing. Georgia Institute of Technology, School of Material Science and Engineering, Atlanta, GA, 1998.

[9] Sypeck D, Wadley HGN. University of Virginia. unpublished research.

[10] Shanley FR. Weight-Strength Analysis of Aircraft Structures. New York: Dover, 1960.

[11] Budiansky B. Harvard University Report, Mech 319. Int J Solids Struct, in press.

[12] Santosa SP, Wierzbicki T. Comput Struct, in press.

[13] Bastawros A, Stone HA, Evans AG. J Heat Transfer, in press.

[14] Lu TJ, Stone HA, Ashby MF. Acta Mater 1998;46:3619-35.

[15] Beals J, Thompson M. J Mater Sci 1997;32:3595-600.

[16] Ashby MF, Seymour CJ, Cebon D. Metal Foams and Honeycombs Database. Granta Design, 1997.

[17] Davies GJ, Zhen Shu. J Mater Sci 1983;18:1899-911.

[18] Thornton PH, Magee CL. Met Trans 1975;6A:1801.

[19] Simone AE, Gibson LJ. Acta Mater 1998;46:3109-23.

[20] Simone AE, Gibson LJ. Acta Mater 1998;46:2139-50.

[21] Sugimura Y, Meyer J, He MY, Bart-Smith H, Grenestedt JL, Evans AG. Acta Mater 1997;45:5345-59.

[22] Bart-Smith H, Bastawros AF, Mumm DR, Evans AG, Sypeck DJ, Wadley HNG. Harvard University, Mech-313. Acta Mater 1998;46:3583-92.

[23] Grenestedt JL. J Mech Phys Solids 1998;46:29-50 (also unpublished results).

[24] He MY, Hutchinson JW. Harvard University Report. MECH 332, 1998.

[25] Weaver PM, Ashby MF. Acta Mater, in press.

[26] Shih D, Boeing Co. 1997, private communication. 
[27] Agarwal BL, Sobel LH. J Aircraft 1972;14:1000-8.

[28] Aeronautical Technology for the Twenty-First Century. National Academy Press, 1992.

[29] Kraynik AM, Reinelt DA. J Colloid Interface Sci 1996;181:511-20.

[30] Renaud M, Giamei A, Priluck J. Materials Research Society Proceedings, in press.

[31] Bastawros A, Evans AG. Acta Mater, in press.

[32] Krizt B, Ashby MF. Cambridge University, Engineering Department. Report C-MATS/TR240, 1998.

[33] Fleck NA. Cambridge University, Engineering Department. unpublished research, 1998.

[34] He MY, Hutchinson JW. ASTM STP 1984;803(1):277-90.

[35] Silva MJ, Gibson LJ. Int J Mech Sci 1997;39:549-63.

[36] Silva MJ, Gibson LJ, Hayes J. Int J Mech 1995;37:1161.

[37] Bejan A. Heat Transfer. New York: Wiley, 1993.

[38] Holman JP. Metric SI, editors. Heat Transfer. New York: McGraw-Hill, 1989.

[39] Jakob M. Trans ASME 1938;60:384-98.

[40] Farrar DJ. J R Aeronaut Soc 1949;53:1041-52.

[41] Gerard G. Minimum Weight Analysis of Compression Structures. New York: New York University Press, 1956.

[42] Thompson JMT, Lewis GM. J Mech Phys Solids 1972;20:101-9.

[43] Gibson LJ. Mater Sci Engng 1994;67:125.

[44] Buckling of Thin-walled Circular Cylinders. 1965, revised 1968. NASA SP-8007, revised.

[45] Sullins RT, Smith GW, Spier EE. Manual for Structural Stability Analysis of Sandwich Plates and Shells. NASA-CR-1457, 1969.

[46] Wierzbicki T, Abramowicz W. Int J Solids Struct 1983;29:3269-88.

[47] Andrews KRG, England GL, Ghani E. Int J Mech Sci 1983;25:687-96.

[48] Kenny LD. Mater Sci Forum 1996;217(222):1883-90.

[49] Schwartz D. 1997, Boeing Co., unpublished information.

[50] Allen HG. Analysis and Design of Structural Sandwich Panels. Oxford: Pergamon, 1969. 